\title{
REVIEWS
}

\section{Nanomedicine strategies to overcome the pathophysiological barriers of pancreatic cancer}

\author{
Pavan P. Adiseshaiah" ${ }^{1}$ Rachael M. Crist ${ }^{1}$, Sara S. Hook ${ }^{2}$ and Scott E. McNeil ${ }^{1}$
}

Abstract | Pancreatic ductal adenocarcinoma (PDAC) is one of the leading causes of cancerrelated deaths. PDAC remains one of the most difficult-to-treat cancers, owing to its unique pathobiological features: a nearly impenetrable desmoplastic stroma, and hypovascular and hypoperfused tumour vessels render most treatment options largely ineffective. Progress in understanding the pathobiology and signalling pathways involved in disease progression is helping researchers to develop novel ways to fight PDAC, including improved nanotechnologybased drug-delivery platforms that have the potential to overcome the biological barriers of the disease that underlie persistent drug resistance. So-called 'nanomedicine' strategies have the potential to enable targeting of the Hedgehog-signalling pathway, the autophagy pathway, and specific RAS-mutant phenotypes, among other pathological processes of the disease. These novel therapies, alone or in combination with agents designed to disrupt the pathobiological barriers of the disease, could result in superior treatments, with increased efficacy and reduced off-target toxicities compared with the current standard-of-care regimens. By overcoming drug-delivery challenges, advances can be made in the treatment of PDAC, a disease for which limited improvement in overall survival has been achieved over the past several decades. We discuss the approaches to nanomedicine that have been pursued to date and those that are the focus of ongoing research, and outline their potential, as well as the key challenges that must be overcome.

${ }^{1}$ Nanotechnology

Characterization Laboratory, Cancer Research Technology Program, Leidos Biomedical Research, Inc., Frederick National Laboratory for Cancer Research, Frederick, Maryland 21702 , USA. ${ }^{2}$ Office of the Director, National Cancer Institute, National Institutes of Health, Bethesda, Maryland 20892, USA

Correspondence to P.P.A pavan.adiseshaiah@ fnlcr.nih.gov
Pancreatic ductal adenocarcinoma (PDAC), often referred to more generally as pancreatic cancer, is an aggressive form of cancer and is the fourth leading cause of cancer-related death in the USA ${ }^{1}$. Cases of PDAC are relatively infrequent compared with other cancers; in 2016, an estimated 53,070 patients will be diagnosed with pancreatic cancer in the USA, compared with 249,260 new cases of breast cancer. Nevertheless, nearly $80 \%$ of patients with PDAC die within a year of diagnosis ${ }^{2}$, whereas more than $80 \%$ of patients with breast cancer survive beyond the first year ${ }^{3}$. Several factors hinder the survival of patients with PDAC. First, few clear clinical signs or symptoms are evident at the early stages of the disease, and early detection is extremely difficult, owing to the absence of disease-specific biomarkers. Second, the symptoms of advanced-stage disease are usually nonspecific, which can further delay diagnosis, resulting in a poor prognosis - the 5-year survival rate among patients with advanced-stage PDAC is less than $5 \%{ }^{4}$. Finally, surgical resection is possible only in about $10-15 \%$ of patients with PDAC, typically owing to the presence of widespread metastases at diagnosis ${ }^{5}$. Moreover, current chemotherapy and radiation treatments are largely ineffective in most patients.

For decades, gemcitabine, a nucleoside analogue of deoxycytidine that blocks DNA replication and several forms of DNA repair, has typically been used as the standard first-line therapy for PDAC ${ }^{6}$. In general, however, therapeutic responses with gemcitabine, either alone or in combination with other cytotoxic agents, have been disappointing for patients with $\mathrm{PDAC}^{7,8}$. This lack of effectiveness is attributed to the poor plasma stability, inefficient cellular uptake, and complex intracellular metabolism of gemcitabine ${ }^{9}$. In addition, cytidine deaminase (CDA) within cells in the blood and liver metabolizes gemcitabine to an inactive difluorodeoxyuridine ( $\mathrm{dFdU}$ ) metabolite, leading to low tissue exposure and rendering the drug largely ineffective? Because of these unfavourable pharmacological properties, gemcitabine is often used at high doses and is administered frequently, in attempts to improve the effectiveness of therapy; however, intensive dosing also 


\section{Key points}

- A nearly impenetrable stroma and hypovascularity limit drug delivery to pancreatic ductal adenocarcinoma (PDAC) cells and thus the effectiveness of treatments, resulting in a very poor prognosis for patients

- Various nanoparticle-based approaches to overcome the biological barriers to drug delivery that are characteristic of pancreatic cancer are being explored in order to improve patient responses and outcomes

- Nanoformulations comprising albumin-bound paclitaxel (nab-paclitaxel) and liposomal irinotecan (MM-398) are approved for the treatment of PDAC based on survival benefits of $1.8-1.9$ months

- Various signalling pathways (for instance, the MAPK/PI3K, Hedgehog, and autophagy cascades), and the KRAS oncogene are involved in the progression of pancreatic cancer and could serve as alternative therapeutic targets

- Strategies to overcome pathophysiological barriers and normalize the tumour matrix (such as Hedgehog-pathway inhibitors) can relieve the solid stress and improve vessel perfusion to increase nanoparticle penetration of the tumour

- Attributes of nanomedicine, such as small size, a high degree of drug encapsulation, and controlled drug release, can improve drug delivery to the tumour by exploiting novel endocytic routes that are independent of membrane transporters

increases the risk of toxic adverse effects. In addition, several cancer-cell intrinsic (for example, genetic) and cancer-cell extrinsic (for example, microenvironmental) factors have been correlated with the poor prognosis of patients treated with gemcitabine: decreased expression of human equilibrative nucleoside transporter 1 (ENT1), which is required for cellular uptake of gemcitabine; attenuated levels of deoxycytidine kinase (dCK), leading to reduced metabolism of the gemcitabine prodrug to form the active metabolite; increased expression of ribonucleotide-diphosphate reductase subunits 1 and 2 (RRM1 and RRM2), which are associated with enhanced resistance to gemcitabine; and epithelial-to-mesenchymal transition (EMT), which suppresses the expression of the ENT1 transporter ${ }^{10,11}$. More recently, the redox-mediated NRF2 pathway has been implicated in gemcitabine resistance in pancreatic cancer cell lines by enhancing the expression of antioxidant response genes ${ }^{12}$.

In 2011, in a trial of the four-drug combination FOLFIRINOX, consisting of 5-fluorouracil, folinic acid (leucovorin), irinotecan, and oxaliplatin, an unprecedented median overall survival of 11 months was reported for patients with metastatic PDAC, compared to 6.8 months with gemcitabine treatment ${ }^{13}$. Unfortunately, the four-drug regimen was associated with substantial increases in the incidence of grades 3-4 toxicities, including neutropenia, diarrhoea, and sensory neuropathy ${ }^{13}$; thus FOLFIRINOX is recommended as a first-line therapy only for patients who are able to endure the toxicities. Several other molecularly targeted therapies have been evaluated as treatments for metastatic PDAC in combination with the standard-of-care treatments, but most have been associated with limited or no clinical benefit, and have failed to progress to advanced stages of clinical evaluation ${ }^{14}$.

The cancer-cell extrinsic pathological features that characterize PDAC, such as the desmoplastic stroma and compressed blood vessels, can also limit the therapeutic effectiveness of treatment ${ }^{15}$. These features impede the delivery of drugs from the circulation to the cancer cells $^{16}(B O X 1)$. These drug-delivery challenges for systemic administration of chemotherapeutic agents, which contribute to the poor effectiveness and overt toxicity of treatment, can be successfully overcome, in part, by exploiting nanotechnology-based strategies - so-called nanomedicines ${ }^{16}$. Such strategies can involve the use of nanoparticle treatments alone or in combination with agents that modify or 'normalize' the tumour stroma (including angiotensin II receptor antagonists, recombinant human hyaluronidase, and inhibitors of Hedgehog signalling), or the tumour vasculature (such as antiangiogenic agents). Normalizing the tumour stroma can reduce solid stress and improve tumour vessel perfusion, thus improving the delivery of the nanoparticles $^{16,17}$. Nanoparticle carriers with appropriately tailored physicochemical characteristics (including optimal size, charge, shape, and drug loading and release), which can protect their cargo (the drug) from premature release and degradation, and can traverse the various biological barriers to drug delivery, have the potential to improve therapeutic efficacy in patients with PDAC (BOX 2). Indeed, advances in nanotechnology have been leveraged for improved drug-delivery strategies for a variety of therapeutic and diagnostic agents ${ }^{18}$. For example, several nanoformulations of gemcitabine have been evaluated in preclinical and clinical studies ${ }^{19-23}$. Furthermore, numerous other nanomaterials are currently undergoing or have completed clinical evaluation for the treatment and/or diagnosis of PDAC specifically (TABLE 1), and these studies have led to new approvals of nanomedicines for PDAC. In this Review, we explore the various signalling pathways and pathological barriers to effective treatment of PDAC, and discuss the nanotechnology-based strategies that are currently being developed to address these challenges and improve therapeutic outcomes for patients with this devastating disease.

\section{Approved nanomedicines for PDAC}

In 2013, the FDA approved an albumin-bound nanoformulation of paclitaxel, nab-paclitaxel, for use in combination with gemcitabine as the frontline treatment for patients with advanced-stage metastatic pancreatic cance $^{24}$. In the phase III clinical trial of this combination $^{24-26}$, a modestly improved 1-year survival rate was demonstrated with the addition of nab-paclitaxel to gemcitabine: $35 \%$ compared with $22 \%$ for gemcitabine alone. Several different mechanisms have been suggested to underlie the therapeutic activity of nab-paclitaxel; for example, the concentration of paclitaxel in the tumour might be increased as a result of active transport mechanisms mediated by the gp60 albumin receptor (albondin) and/or SPARC (also known as secreted protein acidic and rich in cysteine), or scavenging of extracellular proteins, including albumin, by cells in nutrient-deprived tumours in order to sustain growth ${ }^{27-29}$. Other mechanisms that have been proposed for the enhanced treatment outcomes seen when combining nab-paclitaxel with gemcitabine include nab-paclitaxel-mediated inhibition of the gemcitabine-metabolizing enzyme CDA, thereby increasing the intratumoural accumulation of gemcitabine, and/or depletion of the tumour stroma, resulting 


\section{Box 1 | Pathophysiological barriers of PDAC}

- Pancreatic ductal adenocarcinoma (PDAC) arises in an exocrine region of the pancreas and is graded into various stages depending on the histology of the intraepithelial neoplasms (PanlN-1-3); each PanIN stage is associated with unique histological characteristics, and accumulation of mutations at each stage correlates with disease progression ${ }^{130}$

- PDAC is characterized by the presence of a dense desmoplastic stroma, consisting of cellular (endothelial, nerve, and immune cells, and fibroblasts) and acellular (fibrin, collagen, fibronectin, and hyaluronan) components, and has a high ratio of stromal to neoplastic tissue ${ }^{130,131}$; neoplastic cells typically constitute $<20 \%$ of the tumour mass

- The desmoplastic nature of the stroma results in the generation of solid stress and/or increased interstitial fluid pressure within the tumour, leading to vessel compression ${ }^{130,131}$, which in turn, results in inadequate perfusion and hypovascularity, rendering approximately $80 \%$ of the vessels in the tumour nonfunctional ${ }^{17}$

- As a result of inadequate perfusion and hypovascularity, the tumour microenvironment in PDAC is generally hypoxic ${ }^{130,131}$

- Pancreatic stellate cells within the tumour stroma can be activated by inflammatory cytokines, such as IL-1 and IL-6, and growth factors, including tumour necrosis factor $\alpha$ (TNF $\alpha$ ) and transforming growth factor $\beta 1$ (TGF $\beta 1$ ), leading them to secrete copious amounts of extracelleular matrix components that create a barrier to drug extravasation into the tumour interstitium ${ }^{176}$

- Matrix metalloproteinases (MMP-1 and MMP-9) secreted by stellate cells degrade basement membrane proteins, resulting in the initiation of fibrosis and cancer-cell invasion ${ }^{177}$
Thus, enhanced nanoparticle-mediated delivery of potent cytotoxic agents (paclitaxel and irinotecan) to the tumour site have led to improvements in progressionfree survival (PFS) and overall survival for patients with PDAC. The superior performance of the FDA-approved nanomedicines would, however, be best evaluated in direct comparisons of combination regimens comprising the nanoformulation versus similar regimens incorporating the legacy drug (that is, the original, free-drug formulation) - in order to gain insight into whether the improvements in efficacy can simply be explained by the addition of an extra cytotoxic agent to the treatment regimen. Neither the nab-paclitaxel clinical trial, nor the NAPOLI- 1 trial of MM-398 included the free-drug combination treatment (that is, Cremophor EL formulated paclitaxel plus gemicitabine, and free irinotecan plus 5-fluorouracil and folinic acid, respectively) for comparison. Nevertheless, evidence from independent preclinical and clinical studies indicates that the nanoformulations provide an advantage over the legacy formulations by improving the pharmacokinetics of the chemotherapeutic agent. Preclinically, nab-paclitaxel has demonstrated enhanced antitumour activity, increased tumour accumulation, and improved transcytosis (transport across endothelial cells) compared with paclitaxel ${ }^{35}$. Nab-paclitaxel achieved a 33\% higher intratumoural paclitaxel concentration, and endothelial transport was increased compared with that observed with an equal dose of the legacy formulation in mice bearing human breast tumours ${ }^{35}$. Similarly, nanoliposomal irinotecan was associated with enhanced inhibition of tumour growth in comparison with free irinotecan in several preclinical tumour model $s^{36}$. This improved effectiveness was attributed to the 10 -fold enhanced systemic plasma and intratumoural concentrations of irinotecan and its active metabolite, $\mathrm{SN}-38$, compared with those achieved using the legacy irinotecan formulation ${ }^{36}$. In addition, although the results are not directly comparable, an independent randomized clinical trial with the legacy formulation of irinotecan, 5-fluorouracil, and folinic acid (FOLFIRI) in patients with advanced-stage pancreatic cancer reported a median overall survival of only 3.9 month $^{37}$. Of note, however, a median overall survival of 6.0 months was reported in a retrospective analysis of 40 patients with gemcitabine-refractory metastatic PDAC treated with the FOLFIRI regimen ${ }^{38}$. These mixed clinical findings suggest a need for large multicentre randomized trials to further evaluate the efficacy of combination treatments of cytotoxic drugs, and to identify the optimum dosing regimens for patients with metastatic PDAC.

\section{Improving the efficacy of gemcitabine}

In efforts to improve the dismal outcomes of patients with PDAC, gemcitabine has been evaluated clinically in combination with a variety of other treatments, including chemotherapies (such as cisplatin and oxaliplatin), targeted therapies (including erlotinib, cetuximab, and bevacizumab), and a farnesyltransferase inhibitor (tipifarnib) that inhibits RAS proteins, which frequently harbour activating mutations in $\mathrm{PDAC}^{39}$. Only one such treatment has received FDA approval: in 2005, erlotinib 


\section{Box 2 |Advantages of nanomedicine approaches to drug delivery}

- Nanoparticles can be used to increase drug accumulation in tumours by leveraging the enhanced permeation and retention (EPR) effect (passive targeting), whereby the leaky vasculature (gaps in the endothelial walls) and a dysfunctional lymphatic system result in accumulation of circulating nanomedicines in tumour tissue $\mathrm{e}^{173,174}$

- To achieve even greater drug accumulation, nanoparticles can be modified with specific targeting ligands and/or receptors that mediate homing to cognate receptors and/or ligands expressed in the tumour (active targeting), thereby reducing off-target toxicities ${ }^{174,175}$

- Nanoformulations can be exploited to improve the solubility of hydrophobic drugs with unfavourable pharmacokinetics (for example, taxanes), affording a high concentration of drug loading and controlled release of the active pharmaceutical agent

- Nanoformulations can further reduce or eliminate off-target toxicities by removing solubilizing excipients, and have the potential to overcome immunotoxicities associated with siRNA and other oligonucleotide therapies (gene therapy) ${ }^{178,179}$

- Nanoparticles can be masked from immune recognition using surface modifications, such as attachment of polyethylene glycol moieties (PEGylation), to improve systemic circulation and reduce uptake by the mononuclear phagocyte system

- Physicochemical properties of nanoparticles, such as size, electric charge, and hydrophobicity, can be tailored to improve the biodistribution, toxicity profiles, and pharmacokinetic properties of the drug ${ }^{180}$

was approved for use in combination with gemcitabine, although the overall survival of patients treated with this combination was only marginally superior to that of patients in the gemcitabine plus placebo group (median overall survival of 6.2 months versus 5.9 months $)^{40}$.

Cellular uptake of gemcitabine is dependent on ENT1 activity ${ }^{10,41-43}$. In fact, differences in gemcitabine uptake and therapeutic outcomes between patients with PDAC are correlated with variation in the expression of ENT1 (REFS 42,44,45). A gemcitabine-5'-elaidic acid ester conjugate, CP-4126 (previously known as CO-101), was developed to overcome this dependence on ENT1 expression in pancreatic tumours and thereby improve the exposure of tumour cells to gemcitabine ${ }^{46}$ : conjugation with the elaidic acid, an unsaturated fatty acid, increases the lipophilicity of gemcitabine, enabling it to passively enter cells by diffusion, independently of ENT1. Unfortunately, in a randomized phase II trial (LEAP) ${ }^{23}$, CP-4126 treatment did not improve the survival of patients with metastatic PDAC, in comparison to gemcitabine treatment, irrespective of tumour ENT1 expression levels; rapid extracellular metabolism of CP-4126 to gemcitabine, owing to poor stability of the lipid-ester conjugate, has been hypothesized to explain the lack of improvement in overall survival with use of this agent ${ }^{46}$. A phase I trial in patients with solid tumours (including those with PDAC) revealed that the mean initial half-life of gemcitabine formed from CP-4126 was double that of native gemcitabine ( $\mathrm{T}_{1 / 2 \alpha} 15 \mathrm{~min}$ versus $7 \mathrm{~min}$ ), whereas no statistically significant difference in the area under the curve for the dFdU:gemcitabine ratio was demonstrated ${ }^{47}$. These data imply that further improving the half-life of the gemcitabine-lipid conjugate, in addition to controlled release of the drug, could translate to therapeutic benefit in patients with PDAC.

In light of the disappointing result reported for CP-4126, better stratification of patients for gemcitabine treatment, potentially based on ENT1 expression levels, might result in improved effectiveness. Findings of the European Study Group for Pancreatic Cancer ESPAC-3 trial ${ }^{48}$ have suggested that gemcitabine treatment should be reserved for patients with unresected primary tumours expressing high levels of ENT1. A proteomic analysis of PDAC tissues taken from patients who had received gemcitabine, however, did not demonstrate a correlation between ENT1 expression and $\mathrm{PFS}^{49}$, emphasizing the uncertainty of using ENT1 as a marker of the effectiveness of gemcitabine. Indeed, ENT1-independent mechanisms - for example, those related to enzymes involved in gemcitabine metabolism, including dCK, CDA, and RRM1/2 - can also contribute to gemcitabine resistance and might in part explain the limited clinical efficacy of CP-4126. Protein expression levels of dCK, which is involved in the phosphorylation of gemcitabine to form the active monophosphate metabolite, were positively correlated with, and served as a good predictor of, PFS in patients with PDAC treated with gemcitabine ${ }^{49}$.

The effectiveness of gemcitabine treatment is also influenced by the presence of pathophysiological barriers in PDAC, such as the desmoplastic stroma. To understand the correlation between the desmoplastic stroma, ENT1 expression, and incorporation of gemcitabine into DNA, a multiscale transport hypothesis has been proposed ${ }^{41}$. On the basis of mass-transport properties of tumours, derived using CT scans, gemcitabine incorporation into tumour DNA, as determined in resection specimens, was positively correlated with ENT1 levels upon immunohistochemical analysis, and was inversely correlated with stroma scores by Masson's Trichrome staining in patients with PDAC ${ }^{41,42}$. The findings from a clinical trial ${ }^{50}$ reveal parameters, including factors relating to the tumour microenvironment (vascular density, and collagen content of the extracellular matrix (ECM)), and gemcitabine transport (ENT1 expression) that explain the variability in treatment response observed in patients ${ }^{41}$. All of these parameters contribute to tumour heterogeneity, and suggest that a framework of reliable biomarkers of vasculature and stromal densities could help in stratifying the patient population. The stratified patients could potentially be treated with appropriate agents to overcome the pathophysiological barriers and thus enhance nanomedicine delivery. Important efforts are already underway to identify prevalent mutations using next-generation sequencing of DNA from patient samples of PDAC isolated using endoscopic ultrasonography and fine-needle aspiration, in order to stratify patient populations ${ }^{51}$.

On the basis of the clinical success of the nabpaclitaxel plus gemcitabine regimen in patients with PDAC $^{24-26}$, several other nanoformulations of gemcitabine have begun to emerge in the scientific literature. For example, a novel targeted nanomedicine has been developed, comprising a CKAAKN peptide - which mediates efficient homing to pancreatic tumour cells and angiogenic blood vessels via an unknown mechanism - conjugated to squalene and co-nanoprecipitated with a squalenoyl prodrug form of gemcitabine $^{52}$. Treatment with this targeted nanoparticle construct results in improved antitumour responses, as evaluated by enhanced apoptosis, compared with 
Table 1 | Pancreatic cancer clinical trials of nanomedicine strategies for drug delivery

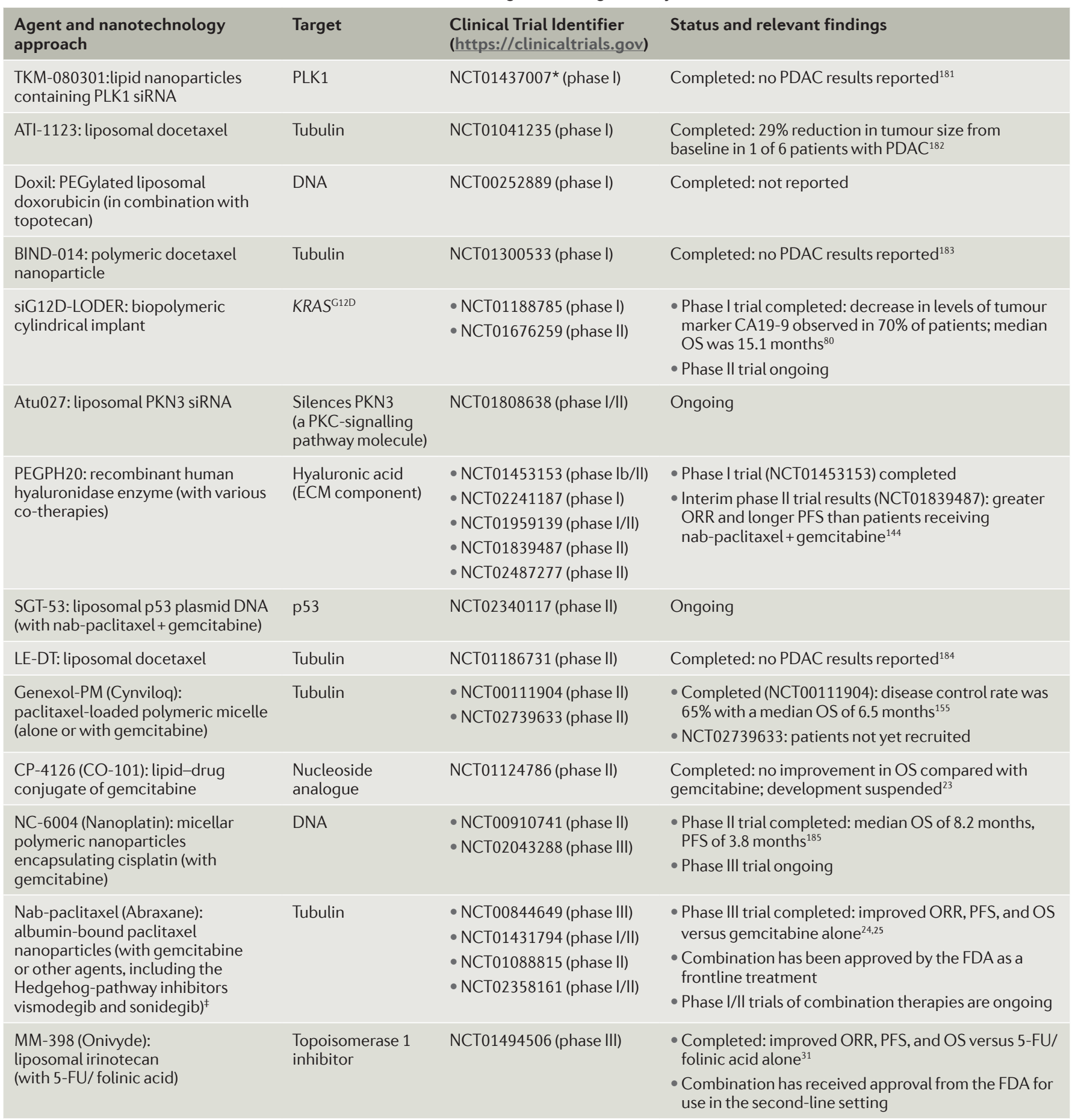

5-FU, 5-fluorouracil; ECM, extracellular matrix; ORR, objective response rate; OS, overall survival; PDAC, pancreatic ductal adenocarcinoma; PEG, polyethylene glycol; PFS, progression-free survival; PKC, protein kinase C; PKN3, protein kinase N3; PLK1, polo-like kinase 1; siG12D-LODER, small-interfering RNA targeting KRASG12D local drug eluter; siRNA, small-interfering RNA. ${ }^{*}$ Trial involved patients with liver cancer or with liver metastases from other cancers (including pancreatic cancer). ${ }^{\ddagger}$ Several ongoing clinical trials are examining various combination treatments; a keyword search of "Abraxane and pancreatic" revealed 190 clinical trials.

those observed with the squalenoyl prodrug of gemcitabine only, or with gemcitabine alone in a RIP-Tag2 mouse model $^{52}$ that recapitulates the multistage tumorigenesis process of pancreatic $\beta$-cell transformation in the islets of Langerhans ${ }^{53}$. In addition, a lipid bilayer mesoporous silica nanoformulation (LB-MSNP) has been developed for simultaneous delivery of gemcitabine and paclitaxel in an optimized synergistic ratio ${ }^{54}$. In a tumour regression study conducted in murine Panc1 xenograft and orthotopic pancreatic cancer models, codelivery of gemcitabine and paclitaxel via the LB-MSNP resulted in superior tumour shrinkage versus that 
observed for LB-MSNP loaded only with gemcitabine, for free gemcitabine, and for free gemcitabine plus nab-paclitaxel ${ }^{54}$. Moreover, high-performance/pressure liquid chromatography analyses of tumour tissues excised following a single intravenous injection of LB-MSNP revealed a 13-fold increase in intratumour levels of active gemcitabine ${ }^{54}$, suggesting the nanoformulation can overcome the pathological barriers to drug delivery, the dependence of this process on the expression of transporters, and the rapid deamination of gemcitabine by CDA. Other examples of nanoformulations of gemcitabine that are under ongoing preclinical development include squalenoyl gemcitabine nanoparticles, which have been shown to passively diffuse into tumour cells independent of the nucleoside transporter ENT1 (REF. 55), and a nanoformulation of gemcitabine and paclitaxel conjugated to a linker that is hydrolysed under mildly acidic conditions ( $\mathrm{pH}$ 6), which should facilitate intracellular release of the drug molecules following endocytosis ${ }^{56}$. In addition, prolonged, controlled drug release has been demonstrated for gemcitabine encapsulated in $130 \mathrm{~nm}$ poly(lactic-co-glycolic acid) (PLGA) polymers or $150 \mathrm{~nm}$ liposomes ${ }^{22}$. Interestingly, treatment of drug-resistant human Pancl cells with PLGAencapsulated gemcitabine resulted in greater induction of cell death and apoptosis in vitro, compared with a liposomal formulation or the free drug ${ }^{22}$. These and other nanoformulations of gemcitabine are currently being optimized in the preclinical setting as treatments for PDAC ${ }^{20,21}$.

\section{Targeting PDAC signalling pathways}

Focus has been placed on understanding the signalling pathways that are activated in the pathogenesis of PDAC in order to identify targets for therapy that might improve clinical outcomes. In PDAC, various signalling pathways, such as the RAS and/or PI3K, and Hedgehog cascades, among others, have established roles in tumorigenesis and disease progression ${ }^{57,58}$ (FIC. 1). Many of these signalling pathways are well studied, and small-molecule inhibitors that target specific effectors involved in transmitting the survival signals are currently in clinical development ${ }^{59-63}$. More recently, another signalling/metabolic pathway has also gained considerable attention as a target for treatments of pancreatic cancer: the autophagy pathway ${ }^{64-66}$.

The RAS-PI3K pathway. The receptor tyrosine kinase (RTK)-signalling pathways that encompass both the RAS-MAPK and PI3K-AKT cascades have an important role in PDAC development and progression. RTK and $\mathrm{PI} 3 \mathrm{~K}$ proteins are not recurrently mutated in PDAC; however, the RAS and PI3K signalling pathways are actively involved in PDAC via other mechanisms ${ }^{58}$. For example, the RAS-MAPK pathway (also referred to as the RAS/RAF/MEK/ERK pathway) is often activated in this disease via binding of growth factors to their cognate $\mathrm{RTK}^{67}$. Growth-factor receptors, such as EGFR and VEGFR, can be overexpressed in PDACs, and signalling via these receptors results in activation of the RAS proteins (FIG. 1). Moreover, $>80 \%$ of patients with pancreatic cancer have activating mutations in codon 12 of the KRAS gene ${ }^{68}$. Mutations in KRAS, particularly of amino acids G12 and/or G13, result in constitutive KRAS activity that is uncoupled from normal signalling fluxes initiated by growth-factor receptors. Activated KRAS is able to signal via downstream effector targets of the MAPK pathway (FIG. 1), and components of the PI3K-AKT, NF-кB, WNT- $\beta$-catenin, Notch, and SMAD pathways. Indeed, the complexity of the crosstalk and feedback loops among these pathways is a major factor in resistance to MAPK-pathway inhibitors ${ }^{69}$. The PI3K pathway is known to regulate various cellular functions, such as angiogenesis, proliferation, metabolism, survival, transcription and protein synthesis, and apoptosis ${ }^{70}$. This pathway is upregulated in several cancers and is one of the critical downstream pathways involved in mediating oncogenic KRAS signalling that results in malignant transformation of pancreatic duct epithelial cells ${ }^{71}$ (FIG. 1).

Overall, more than $90 \%$ of patients with PDAC, as well as $32 \%$ of patients with lung cancer, harbour RAS mutations, leading to dysregulation of RAS-signalling pathways $^{72}$. This highlights the considerable potential to target the RAS pathway and the predominant tumour phenotypes resulting from expression of mutant RAS proteins to combat cancers. This potential remains largely untapped, although interest in targeting RAS directly, and/or in targeting the phenotypes of RASdriven cancers, has resurged ${ }^{73-76}$. Inhibitors have been designed to disrupt post-translational farnesylation or geranylgeranylation (commonly referred to as prenylation) of the cysteine residue in the CAAX motif of RAS proteins, and thereby interfere with the cellular localization and, consequently, the activity of this protein in cell survival, proliferation, migration, and metastasis ${ }^{73,74}$. Unfortunately, two farnesyltransferase inhibitors (lonafarnib and tipifarnib) that had advanced to phase III clinical trials for the treatment of advancedstage PDAC, non-small-cell lung cancer (NSCLC), and colorectal cancer (CRC) failed to show any improvement either alone or in combination with the standard-of-care therapies. This lack of efficacy is attributed to compensatory prenylation mechanisms (via geranylgeranyltransferase) that can maintain the membrane localization of RAS proteins despite farnesyltransferase inhibition ${ }^{73,75-77}$.

Nanotechnology strategies for delivery of shortinterfering RNA (siRNA) that disrupts the production of mutant RAS are being explored. For example, effective delivery of KRAS siRNA using polyethylene glycol (PEG)-poly-L-lysine in combination with a PEGpoly-D,L-lactide nanoformulation of arsenic trioxide has been demonstrated to increase apoptosis of Panc1 cells in vitro and in xenograft models in comparison to either of the nanoparticle agents alone ${ }^{78}$. More recently, a small biodegradable polymeric matrix has been shown to protect encapsulated anti-KRAS ${ }^{\mathrm{G} 12 \mathrm{D}}$ siRNA from premature release and degradation ${ }^{79}$; this nanomedicine, siG12D-LODER, was developed to be inserted into pancreatic tumours to achieve slow and prolonged local release of the siRNA. Proof-of-principle in vivo efficacy of this agent was demonstrated in an orthotopic PDAC model using luciferase-expressing Panc1 cells; 


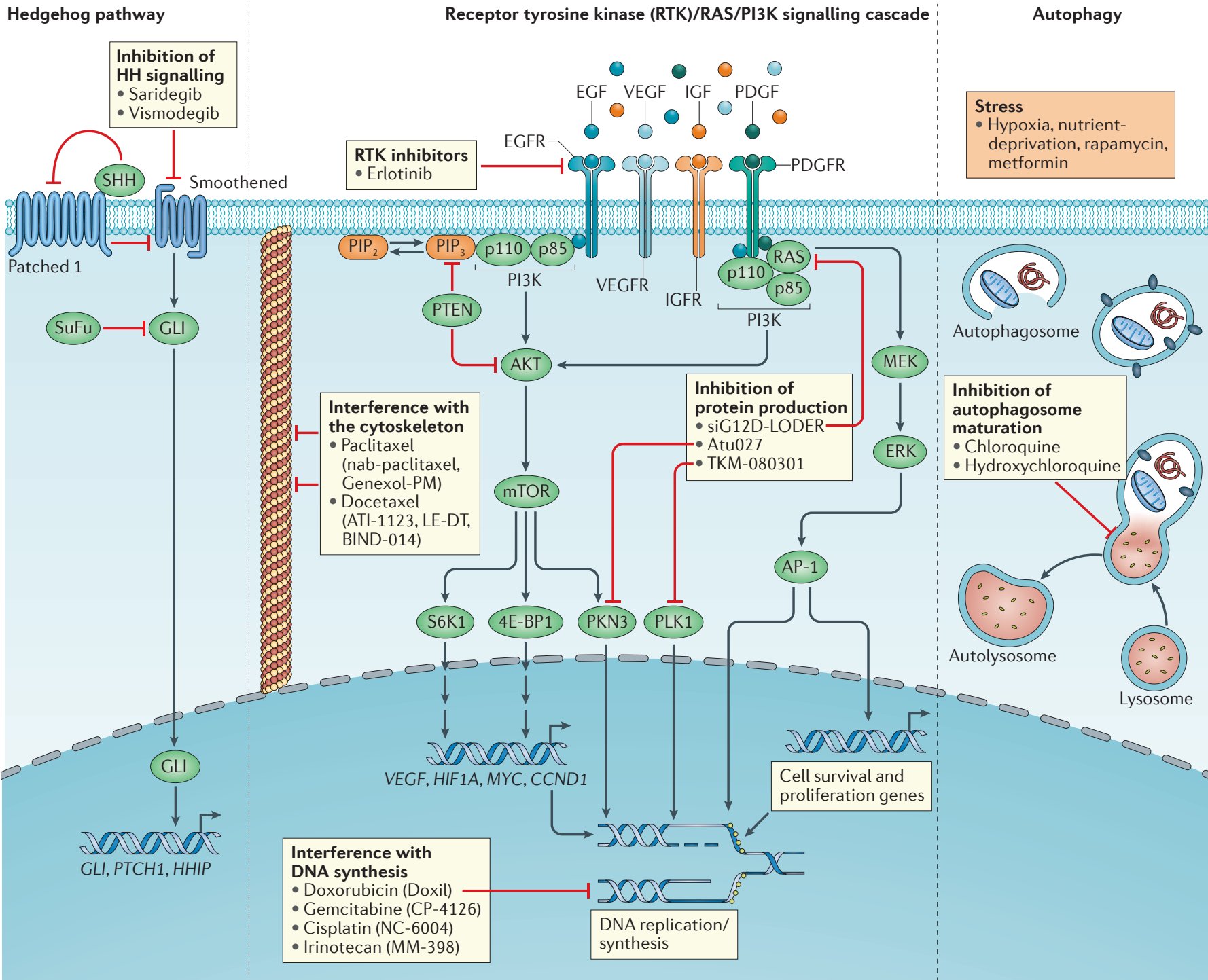

Figure 1 | Critical signalling pathways involved in PDAC pathogenesis. Critical signalling pathways associated with initiation and progression of pancreatic ductal adenocarcinoma (PDAC): from left to right, the Hedgehog $(\mathrm{HH})$, $\mathrm{PI}$ IK/AKT, RAS/MAPK, and autophagy pathways. Each of these pathways can be exploited, independently or in combination, to develop unique treatment strategies. The figure highlights several nanomedicine and/or small-molecule agents, either FDA approved or in various stages of preclinical or clinical development, that are being pursued to inhibit and/or interfere with the pathways involved in PDAC, in order to better combat the disease.

controlled release of anti-KRAS ${ }^{\mathrm{GL2}}$ siRNA from the polymeric matrix was observed, and resulted in prolonged survival compared with that of mice treated with control polymeric particles lacking siRNA. A phase I/IIb clinical study of siG12D-LODER involving 15 patients with locally advanced PDAC has been completed ${ }^{80}$ (TABLE 1). The patients received a single dose of siG12D-LODER, up to $3 \mathrm{mg}(0.375 \mu \mathrm{g}$ particles in 8 capsules), administered near the tumour site using standard endoscopic ultrasonographic guidance procedures ${ }^{80}$; the majority of evaluable patients (10/12) treated with siG12D-LODER had stabilization of tumour growth, and two patients had a partial response to treatment ${ }^{80}$. If the immunological toxicities resulting from exposure to siRNAs (for example, cytokine expression, hypersensitivity, and fever) can be overcome, delivery of these novel therapeutic agents using nanotechnology has tremendous potential to improve on current treatment outcomes. Indeed, the advantage of localized delivery of siG12D-LODER at the tumour site helped to circumvent the immunological toxicities often observed in early phase clinical studies of other systemically administered siRNA nanoformulations ${ }^{81,82}$.

As alternatives to targeting RAS directly, researchers are also developing inhibitors that act on signalling nodes upstream and downstream of RAS. Overexpression of EGFR (also known as ERBB1) is detected in $90 \%$ of patients with PDAC, while overexpression of HER2 (also known as ERBB2) is detected in 21\% of patients with this disease ${ }^{83}$. Overexpression of EGFR and enhanced EGFR signalling is associated with metastasis and decreased 
survival in patients with pancreatic cancer, indicating that targeting the EGFR signalling pathway might provide a survival benefit ${ }^{84}$. Several clinical studies have been conducted to investigate therapies targeting EGFR or VEGFR in patients with advanced-stage solid cancers (including PDAC, CRC, and NSCLC) ${ }^{85-87}$. Unfortunately, for various KRAS-mutated cancer types (NSCLC, PDAC, and CRC), no clinically relevant improvement in patient survival has been observed with the use of inhibitory antibodies (for example, panitumumab and cetuximab for EGFR, and bevacizumab for VEGF) or small-molecules inhibitors (such as the EGFR inhibitors erlotinib and gefitinib) (5-87. $^{\text {. }}$ Of note, although erlotinib in combination with gemcitabine is approved for the treatment of PDAC, the observed improvements in overall survival have been minimal ${ }^{35}$, potentially owing to the very high prevalence of mutations of RAS in these tumours. In a retrospective analysis of KRAS status in patients with PDAC, a median survival benefit of 9.7 months was reported for KRASwild-type patients treated with gemcitabine and erlotinib, compared with only 5.2 months for patients with mutant $K R A S^{88}$. Nevertheless, several alternative nanotechnology strategies that specifically target EGFR or VEGFR are currently being explored, and have been associated with selective drug uptake and improved antitumour activity in several receptor-positive cancer cell lines ${ }^{89,90}$. For example, $\mathrm{O}$-carboxymethyl chitosan (O-CMC)encapsulated, paclitaxel-containing nanoparticles conjugated to the anti-EGFR antibody cetuximab displayed greater activity against EGFR-positive cancer cell lines (A549, A431, and SKBR3 cells) than EGFR-negative cells $\left(\right.$ Mia Paca-2) ${ }^{89}$. In addition, enhanced in vitro anticancer activity was observed with EGFR-targeted nanoparticles, compared with that obtained with nanoparticles lacking cetuximab, suggestive of receptor-mediated selective uptake in EGFR-overexpressing cells ${ }^{89}$. Importantly, targeted nanomedicines such as these hold promise in reducing off-target drug toxicities and improving selective accumulation at the tumour site.

The Hedgehog pathway. The Hedgehog-signalling pathway is primarily involved in morphogenetic signalling during embryogenesis (for example, mediating the development of the pancreatic gland, cell growth, mesenchymal condensation, and cell-fate determination), but has also been shown to be reactivated during the development of PDAC ${ }^{91-93}$. Furthermore, whole-exome sequencing of DNA from microdissected PDAC specimens from 109 patients has revealed frequent genetic alterations affecting the Hedgehog-signalling pathway (such as transition/transversion mutations in the Hedgehog-pathway genes GLI3, SMO, LRP2, GLI2, and $P T C H 1)^{58}$. This pathway comprises three secreted protein ligands, Sonic hedgehog (SHH), Indian hedgehog (IHH), and Desert hedgehog $(\mathrm{DHH})^{94}$. In the absence of ligands, the transmembrane Patched proteins (PTCH1 and PTCH2) inhibit the functionality of a second transmembrane protein, Smoothened (SMO) - via a mechanism that is poorly understood, but is thought to involve sequestration and removal of oxysterol lipid mediators that regulate SMO expression, membrane localization, and/or signalling activity (FIG. 1). Binding of Hedgehog ligands to PTCH1/2 blocks the inhibition of SMO activity, which leads to dissociation of Suppressor of Fused (SuFu)-GLI complexes, enabling nuclear translocation of GLI transcription factors, and thus expression of downstream Hedgehog-signalling target genes ${ }^{95}$. Of note, clinical PDAC specimens that harbour genetic mutations in KRAS and overexpression of EGFR and HER2 commonly have increased mRNA and protein expression of $\mathrm{SHH}$, suggesting activation of the Hedgehog-signalling pathway ${ }^{91}$.

Novel pharmacological strategies are being developed to target the Hedgehog-signalling pathway, in an attempt to disrupt the desmoplastic stroma and enhance drug delivery to cancer cells ${ }^{96}$. A number of smallmolecule inhibitors of Hedgehog-signalling target $\mathrm{SMO}^{97}$, including saridegib (also known as IPI-926), which is a derivative of cyclopamine - a potent SMO inhibitor. When dosed orally in combination with gemcitabine in a transgenic Pdx1-Cre; $\mathrm{Kras}^{+/ \mathrm{LSLG} 12 \mathrm{D}} ; \mathrm{Tp}^{\mathrm{R}} 3^{\mathrm{R} 172 \mathrm{H} /+}$ (KPC) mouse model, saridegib decreased stromal content (levels of collagen I and $\alpha$-smooth muscle actin, and densely packed tumour cells), and increased mean vessel density compared with gemcitabine alone ${ }^{98}$. Indeed, tumours evaluated after 4 days of saridegib-gemcitabine combination treatment exhibited an increase in mean vessel density and CD31 staining, comparable to that observed in non-cancerous pancreatic tissue. These findings have implications for improving the delivery of drugs to pancreatic cancers, particularly for nanoparticle therapies. Saridegib has been evaluated in a phase I/II trial in combination with gemcitabine ${ }^{99}$. This trial was halted, however, owing to poor preliminary results: in the interim analysis, patients who received placebo lived longer in comparison to those given the experimental therapy ${ }^{99}$. Subsequent studies in KPC rodent models demonstrated that saridegib treatment resulted in a more-aggressive cancer phenotype owing to a decrease in the extent of the desmoplastic stroma, leading to decreased survival compared with vehicle-treated mice, mimicking the survival trends observed in $\mathrm{SHH}-$ deficient mice versus control mice ${ }^{100}$. Similarly, poor clinical responses - a low overall response rate, and no improvement in PFS or overall survival - were observed with another SMO inhibitor, vismodegib, in combination with gemcitabine in a phase Ib/II trial in patients with metastatic PDAC ${ }^{101}$. Nevertheless, a second phase I trial investigating saridegib as a combination treatment with FOLFIRINOX is ongoing ${ }^{102}$, based on the rationale that a reduction in the desmoplastic stroma followed by a more-potent combination of cytotoxic drugs might improve the survival of patients with PDAC. Results from a meta-analysis published in 2016 have demonstrated that patients with locally advanced pancreatic cancer treated with FOLFIRINOX had a longer median overall survival (24 months) compared with that of patients who received gemcitabine (6-13 months $)^{103}$, suggesting that combination treatment with saridegib could further improve survival.

Nanotechnologies have been used successfully to improve the delivery of similar Hedgehog-pathway 
inhibitors by overcoming solubility issues, prolonging their systemic half-life, increasing tissue exposure, and thus enhancing treatment responses and reducing toxicity, in a preclinical setting ${ }^{104}$. For example, encapsulation of the hydrophobic SMO inhibitor cyclopamine and the EGFR inhibitor gefitinib using a micellar nanoformulation (methoxy polyethyleneglycol-b-poly-(carbonateco-lactic acid)) resulted in synergistic antitumour activity against the L3.6pl pancreatic cancer cell line, compared with the activity of either agent alone ${ }^{105}$. In addition, a high drug-loading efficiency ( $94 \%$ for cyclopamine) enabled a reduction in the dose of excipients in the formulation ${ }^{105}$. Nanoformulation of HPI-1, an inhibitor that acts on or downstream of GLI via a poorly understood mechanism, using a polymeric platform (NanoHHI) has been shown to improve antitumour responses in an orthotopic pancreatic cancer cell (Pa03C) xenograft model when used in combination with gemcitabine, compared with the use of either of these agents alone ${ }^{104}$. Furthermore, NanoHHI, alone or in combination with gemcitabine, decreased the population of cancer stem cells (CSCs) in this model, as evaluated by analysis of a well-studied marker of this cell type, aldehyde dehydrogenase $(\mathrm{ALDH})^{104}$. This achievement in the preclinical development of novel PDAC treatments is noteworthy, as agents that are effective against CSCs could potentially reduce recurrence of the disease.

Autophagy. Autophagy is a process that involves sequestration of damaged organelles and aggregated proteins into autophagosomes, which subsequently fuse with lysosomes, leading to the degradation and recycling of cellular components in order to maintain cellular homeostasis ${ }^{106}$ (FIG. 1). Autophagy is activated in response to various stressors (such as starvation, viral infection, and different genotoxic agents), and malfunction of this process can lead to the development of several diseases, including cancer ${ }^{107,108}$. Evidence indicates that high levels of autophagy are required to support the development of PDAC ${ }^{65,66}$. Interestingly, results of a study published in 2015 (REF. 109) have demonstrated that the induction of autophagy in PDAC is part of a much larger transcriptional programme, mediated by the microphthalmia/transcription factor E (MiT/TFE) family of transcription factors that coordinates activation of lysosomal biogenesis and function, in addition to nutrient scavenging.

Autophagy has been shown to be upregulated under basal conditions in clinical pancreatic cancer specimens and several pancreatic-cancer cell lines with activating mutations in $K R A S^{66}$, although findings of other studies highlight the complexity in the induction of autophagy ${ }^{110}$. Treatment of cells with the lysosomotropic agent chloroquine blocks the maturation and/or degradation of autophagosomes by inhibiting the fusion of these organelles with lysosomes (FIG. 1), and several studies in preclinical models have demonstrated that abrogating autophagy using chloroquine inhibits cancer-cell growth ${ }^{66,111,112}$. A phase II study of the related agent hydroxychloroquine as a monotherapy ${ }^{113}$, however, failed to show consistent inhibition of autophagy or therapeutic efficacy. Combination with cytotoxic drugs has been proposed as a strategy to overcome the need for complete inhibition of the autophagic response; therefore, chloroquine and the related agent hydroxychloroquine are now being used to target autophagy in combination with cytotoxic agents (such as gemcitabine and nab-paclitaxel) in a number of early phase clinical trials in patients with PDAC ${ }^{66,114-116}$ (for example, NCT01494155 (REF. 117), NCT01506973 (REF. 118), NCT01128296 (REF. 119), NCT01978184 (REF. 120) and NCT01777477 (REF. 121)). The drawbacks of systemically administered chloroquine, however, include the large volumes of distribution and long mean residence time of the drug ${ }^{122}$, owing to sequestration in various tissues ${ }^{123}$, leading to suboptimal efficacy and toxicity upon administration of multiple doses.

By formulating chloroquine within nanoparticles, the pharmacokinetic profile of the drug might be altered to increase its concentration at the tumour site, thus leading to improved efficacy. Several chloroquine-containing nanoformulations have been synthesized and characterized in vitro, but remain at the early, preclinical stages of development ${ }^{124,125}$. Thorough pharmacokinetic analyses, examination of the pharmacodynamic effects on the autophagy pathway (for example, via assessment of autophagic vacuoles and/or microtubule-associated protein 1A/1B-light chain 3 phosphatidylethanolamine conjugate (MAP-LC3-II) accumulation as markers of stalled autophagosome-lysosomal fusion) $)^{126,127}$, and explorations of the in vivo efficacy in multiple pancreaticcancer models are needed to help gauge the therapeutic potential of these novel nanoformulations.

Gemcitabine is a known inducer of autophagy via modulation of transcription factor EB (TFEB), a master regulator of autophagy and lysosomal gene expres$\operatorname{sion}^{128}$, and via early induction of vacuole membrane protein 1 (VMP1) expression, leading to apoptotic cell death $^{129}$. As such, the therapeutic effectiveness of gemcitabine could potentially be increased when used in combination with autophagosome maturation inhibitors (such as chloroquine, hydroxychloroquine, or vinblastine); nanoformulations of gemcitabine with improved pharmacokinetic and pharmacodynamic properties might result in further improvements. Of note, lysosomes are a key intracellular target for modulation of autophagy, and delivering therapeutic agents targeting these organelles selectively to cancer cells without invoking systemic (dose-limiting) toxicity can be particularly challenging. Selectivity can partly be achieved with use of nanoparticles that utilize clathrin-mediated endocytosis, and that include targeting ligands selective to cancer cells ${ }^{106}$.

\section{Overcoming pathobiological barriers}

Important advances have been made in elucidating and deciphering the pathology and genetic factors involved in PDAC tumorigenesis ${ }^{130-132}$. The pathological barriers of PDAC - the desmoplastic stroma, solid stress, interstitial fluid pressure (IFP), and hypovascularity (BOX 1) - can substantially affect the transport of systemic therapies to the tumour cells. Hence, nanoparticle-mediated drug 

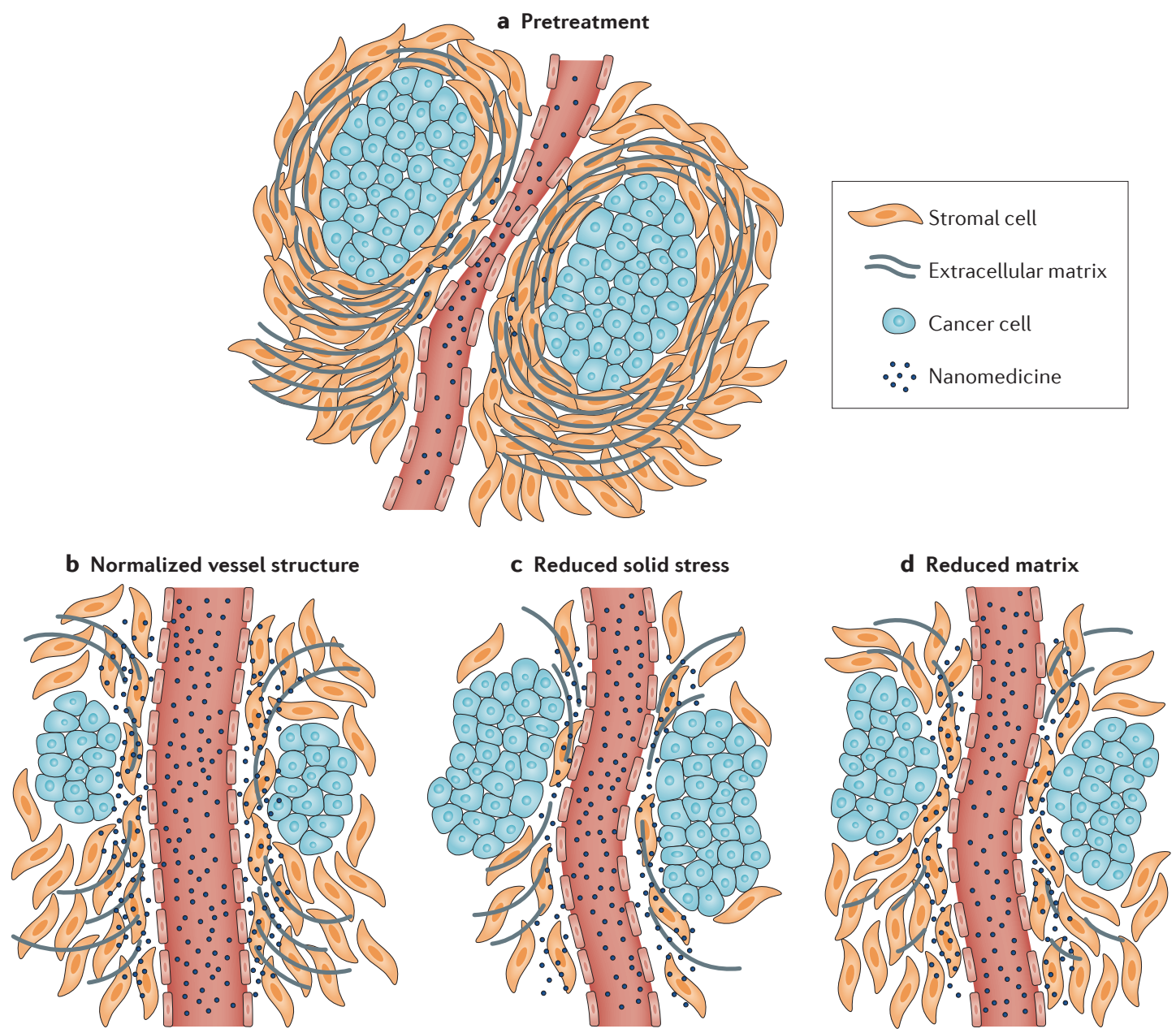

Figure 2 | Strategies to overcome the pathophysiological barriers impeding the effectiveness of treatments of PDAC. a | Pancreatic ductal adenocarcinoma (PDAC) remains extremely difficult to treat, owing to several unique pathobiological barriers that restrict drug delivery to tumour cells: a dense desmoplastic stroma, excessive extracellular matrix deposition, increased interstitial fluid pressure, and compression of blood vessels. Nanomedicine drug delivery can be improved when used in combination with several novel strategies to overcome these pathophysiological barriers. b | Vascular normalization, whereby the tumour vessels become homogeneous, mature and less leaky, and interstitial fluid pressure is reduced, can be achieved using antiangiogenic agents (such as the anti-VEGF antibody bevacizumab). The transient improvement in vessel perfusion increases blood flow and can be exploited to increase nanomedicine delivery to the tumour by convective transport. c | Normalizing the solid stress by reducing the desmoplastic stroma (cellular and acellular components) will increase vessel perfusion, which can enhance the delivery of nanomedicines to the tumour. $\mathbf{d}$ | Reduction of extracellular matrix by depleting the acellular stromal components (such as collagen and hyaluronan) can reduce the interactions of matrix with the nanomedicines and improve the homogeneous distribution in the tumour.

delivery, combined with strategies designed to alleviate the solid stress and/or reduce the IFP within tumours to improve vascular perfusion, have the potential to improve the treatment of PDAC (FIG. 2).

Targeting the stromal compartment. Several strategies to disrupt the physical barrier formed by the desmoplastic stroma that is characteristic of PDAC have been tested, including pharmacological inhibition of the Hedgehog-signalling pathway with saridegib and vismodegib, and systemic administration of PEGylated hyaluronidase (an enzyme that degrades hyaluronan, a major stromal matrix component $)^{98,101,133}$. In two independent studies ${ }^{133,134}$, enzymatic depletion of hyaluronan using PEGylated recombinant human PH20 hyaluronidase (PEGPH20) increased tumour perfusion compared with that observed in untreated tumours, resulting in enhanced tumour delivery of the chemotherapeutic agents doxorubicin and gemcitabine, as quantitated by fluorescence and liquid chromatography-mass spectrometry, respectively. In a separate study, depletion of hyaluronan with PEGPH20 in a KPC mouse model resulted in increased tumour accumulation of fluorophore-conjugated high-molecular-weight dextrans $(40 \mathrm{kDa} \text { and } 2 \mathrm{MDa})^{134}$; enhanced macromolecule accumulation in the tumour tissue was attributed to PEGPH20-induced increases in the size and number of fenestrae and interendothelial gaps, as observed using 
scanning electron microscopy ${ }^{134}$. In fact, both of these strategies for targeting the pancreatic tumour stroma result in modest short-term improvements in the delivery and efficacy of gemcitabine in preclinical models of pancreatic cancer ${ }^{98,99,101,133}$; however, the failure to achieve similar therapeutic improvements in the clinical setting highlights the difficulties in predicting patient responses based on preclinical data ${ }^{101}$. Of note, patients are often found to have a very complex and heterogeneous tumour microenvironment - influenced by both tumour-cell (genetic and epigenetic) and stromal factors - compared with that observed in preclinical models ${ }^{135}$. In addition, the use of animal models with a much simpler disease biology and/or early stage tumours can result in overestimation of improvements in therapeutic efficacy, contributing to the poor preclinical-to-clinical correlation $^{136,137}$. Thus, depletion of the desmoplastic stroma can enhance drug accumulation at the tumour site; however, this approach can also promote cancer-cell proliferation and compromise overall survival, exemplifying the potential advantages and disadvantages of this treatment strategy for PDAC ${ }^{101,134,138}$.

Compelling results obtained through either genetic deletion of SHH or the myofibroblast cell lineage, or pharmacological intervention with PEGPH20 or Hedgehog-pathway inhibitors (saridegib and vismodegib), demonstrate that marked suppression of the desmoplastic stroma increases PDAC aggressiveness in mouse models ${ }^{100,137,139,140}$ - thus elucidating a role of the stroma in restraining tumour growth, rather than in supporting tumorigenesis. Notably, these independent preclinical studies revealed that stromal suppression leads to enhanced tumour invasiveness and, ultimately, worse survival outcomes via increased expression of EMT markers, a greater abundance of undifferentiated cells with CSC-like characteristics, and increased tumour hypoxia ${ }^{100,134,138,141}$. Conversely, findings suggest that activation of the Hedgehog-signalling pathway results in decreased cell proliferation (PanIN epithelial cells and Pdx1-positive progenitor population) and enhanced stromal hyperplasia (increased expression of collagen 1 and $\alpha$ smooth muscle actin) that restrain tumour growth $^{100,137,139,140}$. These findings have been further validated by the results of a retrospective analysis of the stromal content of tumour samples, assessed by co-staining of epithelial tumour cells and the stromal collageneous matrix, from 66 patients with $\mathrm{PDAC}^{142}$ : a high stromal density (defined as a high stromal area to total tumour mass ratio) was associated with a better prognosis and longer patient survival (25.1 months versus 14.4 months for patients with a low stromal density) ${ }^{142}$. Interestingly, depletion of myofibroblasts in transgenic mice with early stage or late-stage pancreatic tumours has been associated with increased CTLA- 4 expression; adminstration of anti-CTLA- 4 antibody markedly attenuated tumour growth and improved survival of the mice by $60 \%{ }^{138}$.

Despite the unexpected role of the desmoplastic stroma in restraining PDAC progression, stromadisrupting agents might provide an avenue for improving drug delivery to pancreatic tumours, including the delivery of large nanomedicines $(>100 \mathrm{~nm}$ in diameter; for example, PEGylated liposomal doxorubicin and MM-398 that have not been associated with therapeutic benefit as monotherapies). Antagonists of Hedgehogsignalling pathways and other interventions (such as PEGPH20) that disrupt the pancreatic tumour stroma have been shown to improve vascularity ${ }^{67,96,97,133}$, which might support increased delivery of cytotoxic agents to the tumour interstitium. Nevertheless, the potency of such combination regimens will require careful modulation to ensure the degree of cancer-cell death caused by the cytotoxic agents counteracts the proliferative effects of stromal disruption on these cells, in order to provide a net improvement in clinical outcome. Of note, Hedgehog-pathway inhibition in combination with gemcitabine chemotherapy was not associated with marked improvements in therapeutic efficacy in preclinical studies and clinical trials, but these findings might be attributable to the poor potency and high metabolic instability of gemcitabine ${ }^{101}$. In a small nonrandomized clinical trial in 59 patients with untreated metastatic PDAC, vismodegib combined with both gemcitabine and nab-paclitaxel was well-tolerated and was associated with a median PFS of 5.5 months and a median overall survival of 10 months ${ }^{139}$, which might be at least partly related to the inclusion of a more-potent cytotoxic agent, nab-paclitaxel, in the treatment regimen.

Similarly, in an ongoing clinical trial ${ }^{143}$, investigators are evaluating the co-administration of gemcitabine, nab-paclitaxel, and PEGPH20. Interim results from this study showed that patients with pancreatic tumours containing high levels of hyaluronan had an improved overall response rate (71\%) and longer median PFS (9.2 months) with the three-agent regimen, compared with nab-paclitaxel plus gemcitabine only (overall response rate of $29 \%$; median PFS of 4.3 months $)^{144}$; however, no substantial differences in the efficacy of these regimens was observed in patients with tumours containing low levels of hyaluronan ${ }^{144}$, suggesting that the benefit of adding PEGPH20 to treatment is dependent on high intratumour levels of hyaluronan.

Treatment with losartan, an angiotensin II receptor antagonist that inhibits production of both stromal collagen and hyaluronan, was shown to result in decompression of tumour blood vessels, improved vessel perfusion, and subsequent allieviation of solid stress in desmoplastic tumour models (4T1 and Pan-02) ${ }^{17,140}$. In the preclinical setting, such depletion of desmoplastic stromal components and improved vessel perfusion enables increased delivery of larger nanoparticles to tumours; for example, by reducing stromal collagen levels, losartan treatment improved the therapeutic efficacy of $100 \mathrm{~nm}$ nanoparticles of PEGylated liposomal doxorubicin (Doxil/Caelyx) ${ }^{140}$. Thus, the lack of efficacy observed with large nanomedicines, such as $100 \mathrm{~nm}$ liposomal doxorubicin, when used as single agents in the clinical treatment of pancreatic cancer might be attributed in part to the solid stress imparted by the desmoplastic stroma ${ }^{145-147}$. Interestingly, in a retrospective analysis of data from patients with advanced-stage pancreatic cancer who were receiving renin-angiotensin pathway inhibitors for the treatment of hypertension, gemcitabine 
therapy improved PFS over that of patients who were not receiving such antihypertensive treatment, which might support the role of these agents in improving gemcitabine delivery to pancreatic tumours ${ }^{148}$. These findings provide compelling evidence that strategies designed to overcome the stromal barriers can open up avenues for efficient delivery of anticancer therapies, including relatively large nanoparticles that carry potent cytotoxic agents, and thereby improve clinical outcomes. A phase II clinical trial of losartan in combination with FOLFIRINOX and radiation therapy in patients with pancreatic cancer is ongoing ${ }^{149}$; however, additional clinical studies of the use of angiotensin-pathway antagonists to improve the efficacy of nanomedicines, specifically, are warranted.

Optimizing nanomedicine to exploit the tumour vasculature. The hypovascular and hypoperfused vasculature of PDAC is leaky owing to the presence of vascular pores (pore sizes vary both within and between tumours), resulting in a heterogeneous blood flow and vascular permeability ${ }^{150}$. In addition, the collapse of the lymphatic network in the centre of the tumour caused by uncontrolled tumour proliferation affects fluid drainage, leading to interstitial hypertension ${ }^{151}$. Besides these physical barriers presented by the hostile tumour microenvironment that hinder drug extravasation, the presence of poorly differentiated regions (with a high vessel density and relatively less stroma) and well-differentiated regions (with a low vessel density and relatively more stroma) in the same tumour can prevent homogeneous distribution of the drug to cancer cells ${ }^{42,100}$. These features all combine to limit the effectiveness of therapy for pancreatic cancer.

In general, the vasculature of pancreatic cancer is moderately permeable, with pore sizes in the range of $50-60 \mathrm{~nm}^{145}$. With this factor in mind, the physicochemical characteristics of nanomedicines - for example, their size - can be tailored to improve the delivery of cytotoxic agents by exploiting the leakiness of the tumour vasculature (FIG. 2). This possibility is exemplified by the enhanced delivery of nab-paclitaxel in comparison to larger nanoparticles (100 nm PEGylated liposomal formulation of doxorubicin, for example). Delivery of nab-paclitaxel to tumours is facilitated by rapid disintegration of the injected $130 \mathrm{~nm}$ nanoparticles in plasma to form smaller particles (approximately $10 \mathrm{~nm}$ in diameter $)^{27,28,152}$. In addition, nab-paclitaxel has a saturable distribution to the peripheral compartment that is approximately ninefold larger than that of the cremophor EL formulation of paclitaxel (1,650 L versus $177 \mathrm{~L})$, contributing to the deep tissue penetration and therapeutic efficacy of nab-paclitaxel ${ }^{153}$. Similarly, Genexol-PM, a 20-50 nm PEG-poly(D,L-lactide) copolymer micellar formulation of paclitaxel, has been associated with improved therapeutic outcomes compared with those of gemcitabine monotherapy in an orthotopic model of gemcitabine-resistant pancreatic cancer ${ }^{154}$. On the basis of these encouraging results, a phase II trial of Genexol-PM has been conducted in patients with locally advanced or metastatic PDAC, with results demonstrating a disease control rate of $65 \%$, and a median overall survival of 6.5 months ${ }^{155}$. A phase II study of
Genexol-PM in combination with gemcitabine ${ }^{156}$ has been initiated in patients with metastatic pancreatic cancer. In another preclinical study, treatment with $20 \mathrm{~nm}$ polymeric micelles encapsulating SN-38 resulted in a similar extent of tumour regression in hypovascular and more desmoplastic tumour (Capan1) models compared with hypervascular and less desmoplastic tumour (PSN1) models ${ }^{157}$; the small size of these nanoparticles enables them to more efficiently extravasate across the moderately permeable vessel walls of PDACs ${ }^{33}$.

In seminal work by Kataoka's group ${ }^{158}$, nanoparticle size was shown to be critical for overcoming pathophysiological barriers to efficient tumour penetration and accumulation in order to improve therapeutic outcomes. Diaminocyclohexane-platinum-based drug micelles of various sizes (30, 50, 70, and $100 \mathrm{~nm}$ ) were evaluated for antitumor efficacy in a hyperpermeable murine colon C26 model (highly vascularized and low stromal content tumours) and a human pancreatic cancer BxPC3 model (poorly vascularized tumours, with reduced permeability and an extensive desmoplastic stroma) $)^{159,160}$. The antitumour activity of the $30 \mathrm{~nm}$ micelles in the BxPC3 model was comparable to that observed with all of the different sized micelles in the hyperpermeable C26 colon tumours; however, the $70 \mathrm{~nm}$ and $100 \mathrm{~nm}$ nanoparticles did not result in tumour regression in the BxPC3 model ${ }^{159,160}$. After fluorescent labelling of $30 \mathrm{~nm}$ and $70 \mathrm{~nm}$ micelles with distinct fluorophores, similar fluorescence intensities and colocalization of particles of both sizes were observed $1 \mathrm{~h}$ post co-injection in mice bearing C26 tumours, confirming efficient extravasation, penetration, and accumulation in hypervascular tumours ${ }^{158}$. In BxPC3 tumour-bearing mice, however, the $30 \mathrm{~nm}$ micelles accumulated in deep tumour tissues, but the $70 \mathrm{~nm}$ micelles remained in close proximity to the vasculature ${ }^{158}$. These study findings further emphasize that small nanoparticles ( $<50 \mathrm{~nm}$ in diameter) are able to more efficiently extravasate and penetrate poorly permeable, hypovascular tumours than larger nanoparticles. Extravasation of larger nanoparticles at the tumour site can be increased in hypovascular and hypopermeable tumours when used in combination with different strategies - that is, agents that improve vascular permeability, or vascular-normalizing agents. For example, a low dose $(1 \mathrm{mg} / \mathrm{kg})$ of the transforming growth factor $\beta$ (TGF $\beta$ ) inhibitor LY364947, which has been shown to temporarily decrease pericyte coverage on the endothelium, improves tumour permeability in the $\mathrm{BxPC} 3$ pancreatic tumour model, resulting in intratumour accumulation of $70 \mathrm{~nm}$ micelles to levels comparable with those observed for the $30 \mathrm{~nm}$ micelles ${ }^{158}$. Similar enhanced accumulation and antitumour activity is observed with $100 \mathrm{~nm}$ liposomal doxorubicin when used in combination with LY364947 (REF. 159).

Thus, nanomedicine approaches to drug delivery can be improved by combining nanoparticle approaches with strategies to overcome the transport barriers that are present in solid tumours; for example, by normalizing either the tumour vasculature using antiangiogenic agents, or the tumour ECM using agents that target the stromal components (such as losartan, PEGPH20, or 
Hedgehog-pathway inhibitors) ${ }^{150}$ (FIG. 2). Agents including the anti-VEGF antibody bevacizumab, and the VEGFR tyrosine kinase inhibitors (TKIs) sunitinib and sorafenib temporally decrease the blood-vessel density and diameters, prune immature tumour vessels, and reduce vascular permeability, thereby converting the tumour vessel architecture to resemble that of normal tissue ${ }^{150,161}$. The improvement in tumour vessel perfusion is, however, dependent on the dose of the antiangiogenic agent used and the tumour type ${ }^{161}$. Treatment with antiangiogenic agents can also reduce the tumoural IFP, which can further improve vessel perfusion, leading to enhanced transport and increased penetration of drugs administered during the 'vessel-normalization window' into deeper regions of the tumour ${ }^{161,162}$.

Three clinical trials ${ }^{86,163,164}$ have been conducted to investigate the ability of agents that target the VEGF pathway (bevacizumab, the 'VEGF trap' aflibercept, and the VEGFR TKI axitinib) to improve the therapeutic efficacy of gemcitabine in patients with advanced-stage PDAC, based on the vascular-normalization concept. In all three trials, adding the antiangiogenic agent to gemcitabine did not improve overall survival ${ }^{86,163,164}$. Despite these disappointing results, some patients $(<5 \%)$ survived more than 2 years after starting combination therapy, suggesting that VEGF-targeted drugs might be of therapeutic benefit to selected patients ${ }^{86,165}$. This finding further signifies the importance of patient stratification to nanoparticle treatments based on the presence of molecular markers of angiogenesis and stromal content, in order to improve clinical outcomes. For example, patients with undifferentiated tumours that have a high vessel density and low levels of stroma might be ideal candidates for antiangiogenic therapy. By reducing the elevated IFP in such tumours, the efficiency of vessels can be increased, and convective transport of large nanoparticles could be improved to achieve deeper tumour tissue penetration (small molecules can diffuse rapidly regardless) ${ }^{100,150}$.

Optimizing nanomedicine to circumvent the ECM. The modest improvements in survival achieved to date through the use of nanomedicines might be influenced by the high binding affinity of nanoparticles to tumour cells or the surrounding ECM. Binding to the ECM can cause the nanoparticles to accumulate in close proximity to the sites of vessel extravasation, which could prevent homogeneous distribution of the drug throughout the entire tumour ${ }^{166}$. In addition to size optimization of the nanoparticles, one must, therefore, consider the ideal surface charge (preferably neutral), which acts to repel or attract components of the interstitial matrix and thereby determine the degree of tumour penetration ${ }^{150}$. Tumours rich in collagen or sulfated glycosaminoglycan can hinder the interstitial transport of charged nanoparticles ${ }^{167,168}$ : collagen fibres are positively charged at neutral $\mathrm{pH}$ and interact with anionic nanoparticles, and negatively charged sulfated glycosaminoglycan fibres or hyaluronan can bind to positively charged nanoparticles to form aggregates, decreasing the interstitial transport and leading to heterogeneous accumulation of nanoparticles ${ }^{167,168}$. Such accumulation can be overcome, to some extent, by including targeting molecules on the surface of nanoparticles that increase the specificity of delivery to tumour cells through receptor-mediated endocytosis, concomitantly reducing nonspecific uptake by normal cells - thus also decreasing the risk of adverse events related to off-target effects. Of note, addition of targeting ligands can also impose further transport limitations within the tumour, owing to the increased particle size and the creation of additional binding sites that can mediate nanoparticle accumulation at certain locations ${ }^{33,150}$. Differences in receptor expression by cancer cells within and across primary and metastatic tumour sites are another important consideration. Despite these limitations, the use of several nanoparticles comprising a targeting moiety, as well as antibody-drug conjugates, has been associated with improved intratumoural penetration of the drug and greater therapeutic efficacy in preclinical and clinical studies ${ }^{169,170}$.

\section{Conclusions}

Patients with PDAC typically have a dismal response to therapy, and despite a considerable increase in our understanding of the various mechanisms that are involved in PDAC development and progression, only modest improvements in patient survival outcomes have been achieved over the past decades ${ }^{171}$. Several challenges limit drug accumulation at the tumour site and/or therapeutic efficacy in patients with pancreatic cancer. First, acquired drug resistance, which can be mediated by induction of drug-detoxification mechanisms (such as CDA expression) $)^{9}$. Second, intrinsic (de novo) drug resistance that can result from a high degree of genomic instability and heterogeneity within both primary and metastatic tumours, and mutations in components of several key functional pathway. Third, hypovascularity and a dense desmoplastic stroma that create barriers that restrict drug delivery to the tumour site ${ }^{138,150,172}$.

PDAC presents a host of pathological features that limit the therapeutic response to many treatments (BOX 1), but nanotechnology-based drug delivery offers alternative strategies for treating this devastating disease. Anticancer nanomedicines have advantages over small-molecule drugs; for example, nanoparticles often preferentially accumulate in tumours owing to the enhanced permeation and retention (EPR) effect ${ }^{173,174}$ (BOX 2). Over the past decade, nanomedicines have entered clinical trials for a range of indications, including pancreatic cancer. The selected studies described herein clearly emphasize the importance of understanding the tumour biology in tailoring nanomedicines to improve on the therapeutic responses observed with standard therapies and achieve clinical translation. Firstgeneration nanomedicines, such as nab-paclitaxel and MM-398, have already been applied to modestly improve the outcomes of pancreatic cancer treatments, and many more novel nanotechnology strategies are being explored in both clinical and preclinical settings. By overcoming the limitations of conventional drug delivery, nanotechnology has the potential to improve the delivery of drugs to the pancreatic-tumour interstitium, and then exploit endocytic or surface receptors to enter tumour cells. 
Furthermore, by including targeting moieties (such as arginylglycylaspartic acid (RGD) motifs, $\alpha_{v}$ integrins, or pentagastrin), nanoparticle internalization by endothelial and pancreatic cancer cells might be further increased.

Many of the nanotechnology strategies described herein can provide a modest, but statistically significant, improvement in the survival of patients with PDAC. Nanomedicine formulation strategies have been demonstrated to increase drug uptake, decrease stromal content and reduce toxicity, emphasizing the potential of nanotechnology-based approaches to circumvent the natural pathological barriers that have hindered PDAC treatment for decades. For improved internalization and distribution of nanoparticles in the tumour, nanoparticles should have a smaller size (for better penetration), contain an agent with high cytotoxic potency, enable controlled drug release, increase the systemic half-life of the agent, and bind with only low affinity to the ECM $\mathrm{E}^{33,150,175}$. Even with fine-tuning and manipulation of the properties of the nanomedicine, the desmoplastic stroma and hypovascularity of patients with PDAC continue to hinder the efficient delivery of nanoformulated drugs in sufficient concentrations to substantially improve patient responses and survival rates. To achieve further therapeutic advantages, appropriately tailored nanomedicines should cause a shift in the balance between the antitumour effects of the cytotoxic agents and the protumorigenic environment that prevails upon depletion of the tumour stroma. Several clinical trials (TABLE 1), as well as ongoing preclinical efforts, offer hope that nanotechnology will soon create new treatment options for the thousands of patients with PDAC around the world.
1. Siegel, R. L., Miller, K. D. $₫$ Jemal, A. Cancer statistics, 2016. CA Cancer J. Clin. 66, 7-30 (2016).

2. National Cancer Institute. A snapshot of pancreatic cancer. http://www.cancer.gov/research/progress/ snapshots/pancreatic (2014)

3. National Cancer Institute. A snapshot of breast cancer http://www.cancer.gov/research/progress/snapshots/ breast (2014).

4. Hidalgo, M. Pancreatic cancer. N. Engl. J. Med. 362 , 1605-1617 (2010)

5. Patra, C. R., Bhattacharya, R., Mukhopadhyay, D. \& Mukherjee, P. Fabrication of gold nanoparticles for targeted therapy in pancreatic cancer. Adv. Drug Deliv. Rev. 62, 346-361 (2010)

6. Burris, H. A. et al. Improvements in survival and clinical benefit with gemcitabine as first-line therapy for patients with advanced pancreas cancer: a randomized trial. J. Clin. Oncol. 15, 2403-2413 (1997).

7. Gresham, G. K., Wells, G. A., Gill, S., Cameron, C ¿ Jonker, D. J. Chemotherapy regimens for advanced pancreatic cancer: a systematic review and network meta-analysis. BMC Cancer 14, 471 (2014).

8. Ihse, I. et al. [Guidelines for management of patients with pancreatic cancer]. Lakartidningen 99 , 1676-1680, 1683-1685 (2002).

9. Shipley, L. A. et al. Metabolism and disposition of gemcitabine, and oncolytic deoxycytidine analog, in mice, rats, and dogs. Drug Metab. Dispos. 20, 849-855 (1992)

10. de Sousa Cavalcante, L. \& Monteiro, G. Gemcitabine: metabolism and molecular mechanisms of action, sensitivity and chemoresistance in pancreatic cancer. Eur. J. Pharmacol. 741C, 8-16 (2014).

11. Zheng, X. et al. Epithelial-to-mesenchymal transition is dispensable for metastasis but induces chemoresistance in pancreatic cancer. Nature $\mathbf{5 2 7}$. 525-530 (2015)

12. Ju, H. Q. et al. Mechanisms of overcoming intrinsic resistance to gemcitabine in pancreatic ductal adenocarcinoma through the redox modulation. Mol. Cancer Ther. 14, 788-798 (2015).

13. Conroy, T. et al. FOLFIRINOX versus gemcitabine for metastatic pancreatic cancer. N. Engl. J. Med. 364 1817-1825 (2011)

14. Zagouri, F. et al. Molecularly targeted therapies in metastatic pancreatic cancer: a systematic review. Pancreas 42, 760-773 (2013).

15. Xie, D. \& Xie, K. Pancreatic cancer stromal biology and therapy. Genes Dis. 2, 133-143 (2015)

16. Stylianopoulos, T. \& Jain, R. K. Combining two strategies to improve perfusion and drug delivery in solid tumors. Proc. Natl Acad. Sci. USA 110 18632-18637 (2013)

17. Chauhan, V. P. et al. Angiotensin inhibition enhances drug delivery and potentiates chemotherapy by decompressing tumour blood vessels. Nat. Commun. 4, 2516 (2013).

18. de Souza, P. C., Ranjan, A. \& Towner, R. A Nanoformulations for therapy of pancreatic and liver cancers. Nanomed. (Lond.) 10, 1515-1534 (2015).

19. Celia, C., Cosco, D., Paolino, D. \& Fresta, M. Gemcitabine-loaded innovative nanocarriers versus GEMZAR: biodistribution, pharmacokinetic features and in vivo antitumor activity. Expert Opin. Drug Deliv. 8, 1609-1629 (2011)

20. Lee, G. Y. et al. Theranostic nanoparticles with controlled release of gemcitabine for targeted therapy and MRI of pancreatic cancer. ACS Nano 7, 2078-2089 (2013)

21. Martin-Banderas, L. et al. Biocompatible gemcitabinebased nanomedicine engineered by flow focusing for efficient antitumor activity. Int. J. Pharm. 443 103-109 (2013).

22. Papa, A. L. et al. Mechanistic studies of gemcitabine loaded nanoplatforms in resistant pancreatic cancer cells. BMC Cancer 12, 419 (2012).

23. Poplin, E. et al. Randomized, multicenter, phase II study of CO-101 versus gemcitabine in patients with metastatic pancreatic ductal adenocarcinoma: including a prospective evaluation of the role of hENT 1 in gemcitabine or CO-101 sensitivity. J. Clin. Oncol. 31, 4453-4461 (2013).

24. Von Hoff, D. D. et al. Increased survival in pancreatic cancer with nab-paclitaxel plus gemcitabine. N. Engl. J. Med. 369, 1691-1703 (2013).

25. Goldstein, D. et al. nab-Paclitaxel plus gemcitabine for metastatic pancreatic cancer: long-term survival from a phase III trial. J. Natl Cancer Inst. 107, dju413 (2015).

26. Neesse, A., Michl, P., Tuveson, D. A. \& Ellenrieder, V. nab-Paclitaxel: novel clinical and experimental evidence in pancreatic cancer. Z. Gastroenterol. 52, 360-366 (2014).

27. Frese, K. K. et al. nab-Paclitaxel potentiates gemcitabine activity by reducing cytidine deaminase levels in a mouse model of pancreatic cancer. Cancer Discov. 2, 260-269 (2012).

28. Kamphorst, J. J. et al. Human pancreatic cancer tumors are nutrient poor and tumor cells actively scavenge extracellular protein. Cancer Res. 75, 544-553 (2015).

29. Von Hoff, D. D. et al. Gemcitabine plus nab-paclitaxel is an active regimen in patients with advanced pancreatic cancer: a phase I/II trial. J. Clin. Oncol. 29 , 4548-4554 (2011)

30. Hoffman, R. M \& Bouvet, M. Nanoparticle albumin bound-paclitaxel: a limited improvement under the current therapeutic paradigm of pancreatic cancer. Expert Opin. Pharmacother. 16, 943-947 (2015).

31. Wang-Gillam, A. et al. Nanoliposomal irinotecan with fluorouracil and folinic acid in metastatic pancreatic cancer after previous gemcitabine-based therapy (NAPOLI-1): a global, randomised, open-label, phase 3 trial. Lancet, 387 545-557 (2015).

32. Chen, L. T. et al. Expanded analyses of napoli-1: Phase 3 study of MM-398 (nal-IRI), with or without 5-fluorouracil and leucovorin, versus 5-fluorouracil and leucovorin, in metastatic pancreatic cancer (mPAC) previously treated with gemcitabine-based therapy [abstract 234]. J. Clin. Oncol. 33 (Suppl. 3) (2015)

33. Stylianopoulos, T. \& Jain, R. K. Design consideration for nanotherapeutics in oncology. Nanomedicine $\mathbf{1 1}$ 1893-1907 (2015)

34. Von Hoff, D. et al. NAPOLI-1: randomized phase 3 study of MM-398 (NAL-IRI) with or without

5-fluorouracil and leucovorin, versus 5-fluorouracil and leucovorin, in metastatic pancreatic cancer progressed on or following gemcitabine-based therapy [abstract O-0003]. Ann. Oncol. 25, ii105-ii 106 (2014).

35. Desai, N. et al. Increased antitumor activity, intratumor paclitaxel concentrations, and endothelial cell transport of cremophor-free, albumin-bound paclitaxel, ABI-007, compared with cremophor-based paclitaxel. Clin. Cancer Res. 12, 1317-1324 (2006).

36. Kalra, A. V. et al. Preclinical activity of nanoliposomal irinotecan is governed by tumor deposition and intratumor prodrug conversion. Cancer Res. 74, 7003-7013 (2014)

37. Yoo, C. et al. A randomised phase II study of modified FOLFIRI. 3 versus modified FOLFOX as second-line therapy in patients with gemcitabine-refractory advanced pancreatic cancer. Br. J. Cancer 101 1658-1663 (2009).

38. Gebbia, V. et al. Irinotecan plus bolus/infusional 5-fluorouracil and leucovorin in patients with pretreated advanced pancreatic carcinoma: a multicenter experience of the Gruppo Oncologico Italia Meridionale. Am. J. Clin. Oncol. 33, 461-464 (2010)

39. Oberstein, P. E. \& Olive, K. P. Pancreatic cancer: why is it so hard to treat? Therap Adv. Gastroenterol. 6 321-337 (2013)

40. Moore, M. J. et al. Erlotinib plus gemcitabine compared with gemcitabine alone in patients with advanced pancreatic cancer: a phase III trial of the National Cancer Institute of Canada Clinical Trials Group. J. Clin. Oncol. 25, 1960-1966 (2007).

41. Koay, E. J. et al. Transport properties of pancreatic cancer describe gemcitabine delivery and response. J. Clin. Invest. 124, 1525-1536 (2014)

42. Koay, E. J. et al. Intra-tumoral heterogeneity of gemcitabine delivery and mass transport in human pancreatic cancer. Phys. Biol. 11, 065002 (2014).

43. Lee, Y. et al. Human equilibrative nucleoside transporter-1 knockdown tunes cellular mechanics through epithelial-mesenchymal transition in pancreatic cancer cells. PLOS ONE 9, e 107973 (2014).

44. Farrell, J. J et al. Human equilibrative nucleoside transporter 1 levels predict response to gemcitabine in patients with pancreatic cancer. Gastroenterology 136, 187-195 (2009).

45. Spratlin, J. L. \& Mackey, J. R. Human equilibrative nucleoside transporter 1 (hENT1) in pancreatic adenocarcinoma: towards individualized treatment decisions. Cancers (Basel) 2, 2044-2054 (2010).

46. Stuurman, F. E. et al. A phase 1 comparative pharmacokinetic and cardiac safety study of two intravenous formulations of CO-101 in patients with advanced solid tumors. J. Clin. Pharmacol. 53 878-883 (2013)

47. Venugopal, B. et al. A first-in-human phase I and pharmacokinetic study of CP-4126 (CO-101), a nucleoside analogue, in patients with advanced solid tumours. Cancer Chemother. Pharmacol. 76, 785-792 (2015)

48. Greenhalf, W. et al. Pancreatic cancer hENT 1 expression and survival from gemcitabine in patients from the ESPAC-3 trial. J. Natl Cancer Inst. 106. djt347 (2014). 
49. Ohmine, K. et al. Quantitative targeted proteomics of pancreatic cancer: deoxycytidine kinase protein level correlates to progression-free survival of patients receiving gemcitabine treatment. Mol. Pharm. 12, 3282-3291 (2015).

50. US National Library of Medicine. ClinicalTrials.gov, https://clinicaltrials.gov/ct2/show/NCT01276613? term $=$ nct01276613\&rank =1 (2011).

51. Gleeson, F. C. et al. Targeted next generation sequencing of endoscopic ultrasound acquired cytology from ampullary and pancreatic adenocarcinoma has the potential to aid patient stratification for optimal therapy selection. Oncotarget http://dx.doi.org/10.18632/oncotarget.9440 (2016).

52. Valetti, S. et al. Peptide-functionalized nanoparticles for selective targeting of pancreatic tumor. J. Control Release 192, 29-39 (2014).

53. Hanahan, D. Heritable formation of pancreatic $\beta$-cell tumours in transgenic mice expressing recombinant insulin/simian virus 40 oncogenes. Nature 315 115-122 (1985)

54. Meng, H. et al. Use of a lipid-coated mesoporous silica nanoparticle platform for synergistic gemcitabine and paclitaxel delivery to human pancreatic cancer in mice. ACS Nano 9, 3540-3557 (2015).

55. Rejiba, S. et al. Squalenoyl gemcitabine nanomedicine overcomes the low efficacy of gemcitabine therapy in pancreatic cancer. Nanomedicine 7, 841-849 (2011)

56. Aryal, S., Hu, C. M. \& Zhang, L. Combinatorial drug conjugation enables nanoparticle dual-drug delivery. Small 6, 1442-1448 (2010).

57. Furukawa, T. Molecular targeting therapy for pancreatic cancer: current knowledge and perspectives from bench to bedside. J. Gastroenterol. 43, 905-911 (2008).

58. Witkiewicz, A. K. et al. Whole-exome sequencing of pancreatic cancer defines genetic diversity and therapeutic targets. Nat. Commun. 6, 6744 (2015).

59. Falasca, M., Selvaggi, F., Buus, R., Sulpizio, S \& Edling, C. E. Targeting phosphoinositide 3-kinase pathways in pancreatic cancer - from molecular signalling to clinical trials. Anticancer Agents Med. Chem. 11, 455-463 (2011).

60. Porta, C., Paglino, C. \& Mosca, A. Targeting PI3K/Akt/ mTOR signaling in cancer. Front. Oncol. 4, 64 (2014).

61. Zhao, Y. \& Adjei, A. A. The clinical development of MEK inhibitors. Nat. Rev. Clin. Oncol. 11, 385-400 (2014).

62. Rosow, D. E. et al. Sonic Hedgehog in pancreatic cancer: from bench to bedside, then back to the bench. Surgery 152, S19-S32 (2012).

63. Collins, M. A. \& Pasca di Magliano, M. Kras as a key oncogene and therapeutic target in pancreatic cancer Front. Physiol. 4, 407 (2013).

64. De Duve, C. The lysosome. Sci. Am. 208, 64-72 (1963).

65. Yang, A. et al. Autophagy is critical for pancreatic tumor growth and progression in tumors with p53 alterations. Cancer Discov. 4, 905-913 (2014).

66. Yang, S. et al. Pancreatic cancers require autophagy for tumor growth. Genes Dev. 25, 717-729 (2011).

67. McCubrey, J. A. et al. Roles of the Raf/MEK/ERK pathway in cell growth, malignant transformation and drug resistance. Biochim. Biophys. Acta 1773, 1263-1284 (2007)

68. Hruban, R. H et al. K-Ras oncogene activation in adenocarcinoma of the human pancreas. A study of 82 carcinomas using a combination of mutant-enriched polymerase chain reaction analysis and allele-specific oligonucleotide hybridization. Am. J. Pathol. 143 545-554 (1993)

69. Ramos, J. W. The regulation of extracellular signalregulated kinase (ERK) in mammalian cells. Int. J. Biochem. Cell Biol. 40, 2707-2719 (2008)

70. Hemmings, B. A. \& Restuccia, D. F. PI3K-PKB/Akt pathway. Cold Spring Harb. Perspect. Biol. 4, a011189 (2012).

71. Campbell, P. M. et al. K-Ras promotes growth transformation and invasion of immortalized human pancreatic cells by Raf and phosphatidylinositol 3-kinase signaling. Cancer Res. 67, 2098-2106 (2007).

72. Cox, A. D., Fesik, S. W., Kimmelman, A. C., Luo, J. ¿ Der, C. J. Drugging the undruggable RAS: Mission possible? Nat. Rev. Drug Discov. 13, 828-851 (2014).

73. Berndt, N., Hamilton, A. D. \& Sebti, S. M. Targeting protein prenylation for cancer therapy. Nat. Rev. Cancer 11, 775-791 (2011).

74. Hancock, J. F., Magee, A. I., Childs, J. E.

\& Marshall, C. J. All ras proteins are polyisoprenylated but only some are palmitoylated. Cell 57, 1167-1177 (1989).
75. Rao, S. et al. Phase III double-blind placebo-controlled study of farnesyl transferase inhibitor R115777 in patients with refractory advanced colorectal cancer. J. Clin. Oncol. 22, 3950-3957 (2004).

76. Riely, G. J. et al. A phase II trial of salirasib in patients with lung adenocarcinomas with KRAS mutations. J. Thorac Oncol. 6, 1435-1437 (2011).

77. Van Cutsem, E. et al. Phase III trial of gemcitabine plus tipifarnib compared with gemcitabine plus placebo in advanced pancreatic cancer. J. Clin. Oncol. 22, 1430-1438 (2004).

78. Zeng, L. et al. Combination of siRNA-directed Kras oncogene silencing and arsenic-induced apoptosis using a nanomedicine strategy for the effective treatment of pancreatic cancer. Nanomedicine 10 463-472 (2014).

79. Khvalevsky, E. Z. et al. Mutant KRAS is a druggable target for pancreatic cancer. Proc. Natl Acad. Sci. USA 110, 20723-20728 (2013).

80. Golan, T. et al. RNAi therapy targeting KRAS in combination with chemotherapy for locally advanced pancreatic cancer patients. Oncotarget 6 24560-24570 (2015)

81. Schultheis, B. et al. First-in-human phase I study of the liposomal RNA interference therapeutic Atu027 in patients with advanced solid tumors. J. Clin. Oncol. 32, 4141-4148 (2014).

82. Zuckerman, J. E. \& Davis, M. E. Clinical experiences with systemically administered siRNA-based therapeutics in cancer. Nat. Rev. Drug Discov. 14 843-856 (2015)

83. Gracián, A. C., Pascual, J. R. \& Medina, M. H. Epidermal growth factor receptor as a target in pancreatic cancer. Cancer Chemother. Rev. 3 223-229 (2008).

84. Kelley, R. K. \& Ko, A. H. Erlotinib in the treatment of advanced pancreatic cancer. Biologics 2, 83-95 (2008)

85. Han, S. W. et al. Optimization of patient selection for gefitinib in non-small cell lung cancer by combined analysis of epidermal growth factor receptor mutation, K-ras mutation, and Akt phosphorylation. Clin. Cancer Res. 12, 2538-2544 (2006)

86. Kindler, H. L. et al. Gemcitabine plus bevacizumab compared with gemcitabine plus placebo in patients with advanced pancreatic cancer: phase III trial of the Cancer and Leukemia Group B (CALGB 80303). J. Clin. Oncol. 28, 3617-3622 (2010)

87. Peeters, M. et al. Mutant KRAS codon 12 and 13 alleles in patients with metastatic colorectal cancer: assessment as prognostic and predictive biomarkers of response to panitumumab. J. Clin. Oncol. 31, 759-765 (2013).

88. Kim, S. T. et al. Impact of KRAS mutations on clinical outcomes in pancreatic cancer patients treated with first-line gemcitabine-based chemotherapy. Mol. Cancer Ther. 10, 1993-1999 (2011).

89. Maya, S. et al. Cetuximab conjugated O-carboxymethyl chitosan nanoparticles for targeting EGFR overexpressing cancer cells. Carbohydr. Polym. 93, 661-669 (2013).

90. Xiangbao, Y. et al. Humanized anti-VEGFR-2 ScFv-As2O3-stealth nanoparticles, an antibody conjugate with potent and selective anti-hepatocellular carcinoma activity. Biomed. Pharmacother. 68 597-602 (2014)

91. Thayer, S. P. et al. Hedgehog is an early and late mediator of pancreatic cancer tumorigenesis. Nature 425, 851-856 (2003)

92. Nusslein-Volhard, C. \& Wieschaus, E. Mutations affecting segment number and polarity in Drosophila. Nature 287, 795-801 (1980).

93. Hebrok, M. Hedgehog signaling in pancreas development. Mech. Dev. 120, 45-57 (2003).

94. Heretsch, P., Tzagkaroulaki, L. \& Giannis, A Modulators of the hedgehog signaling pathway. Bioorg. Med. Chem. 18, 6613-6624 (2010).

95. Pan, Y., Bai, C. B., Joyner, A. L. \& Wang, B. Sonic hedgehog signaling regulates $\mathrm{Gli} 2$ transcriptional activity by suppressing its processing and degradation. Mol. Cell. Biol. 26, 3365-3377 (2006).

96. Onishi, H. \& Katano, M. Hedgehog signaling pathway as a new therapeutic target in pancreatic cancer. World J. Gastroenterol. 20, 2335-2342 (2014).

97. Stanton, B. Z. \& Peng, L. F. Small-molecule modulators of the Sonic Hedgehog signaling pathway. Mol. Biosyst 6, 44-54 (2010).

98. Olive, K. P. et al. Inhibition of Hedgehog signaling enhances delivery of chemotherapy in a mouse model of pancreatic cancer. Science 324, 1457-1461 (2009).
99. Herter-Sprie, G. S., Kung, A. L. \& Wong, K. K. New cast for a new era: preclinical cancer drug development revisited. J. Clin. Invest. 123, 3639-3645 (2013).

100. Rhim, A. D. et al. Stromal elements act to restrain, rather than support, pancreatic ductal adenocarcinoma. Cancer Cell 25, 735-747 (2014).

101. Catenacci, D. V. et al. Randomized phase lb/ll study of gemcitabine plus placebo or vismodegib, a hedgehog pathway inhibitor, in patients with metastatic pancreatic cancer. J. Clin. Oncol. 33, 4284-4292 (2015).

102. US National Library of Medicine. ClinicalTrials.gov, https://clinicaltrials.gov/ct2/show/NCT01383538? term $=$ nct01383538\&rank $=1$ (2011)

103. Suker, M. et al. FOLFIRINOX for locally advanced pancreatic cancer: a systematic review and patient-level meta-analysis. Lancet Oncol. 17, 801-810 (2016).

104. Chenna, V. et al. A polymeric nanoparticle encapsulated small-molecule inhibitor of Hedgehog signaling (NanoHHI) bypasses secondary mutational resistance to Smoothened antagonists. Mol. Cancer Ther. 11 165-173 (2012).

105. Chitkara, D. et al. Micellar delivery of cyclopamine and gefitinib for treating pancreatic cancer. Mol. Pharm. 9 , 2350-2357 (2012)

106. Stern, S. T., Adiseshaiah, P. P. \& Crist, R. M. Autophagy and lysosomal dysfunction as emerging mechanisms of nanomaterial toxicity. Part. Fibre Toxicol. 9, 20 (2012).

107. Ravikumar, B. et al. Regulation of mammalian autophagy in physiology and pathophysiology. Physiol. Rev. 90, 1383-1435 (2010)

108. White, E. \& DiPaola, R. S. The double-edged sword of autophagy modulation in cancer. Clin. Cancer Res. 15, 5308-516 (2009).

109. Perera, R. M. et al. Transcriptional control of autophagy-lysosome function drives pancreatic cancer metabolism. Nature 524, 361-365 (2015)

110. Eng, C. H. et al. Macroautophagy is dispensable for growth of KRAS mutant tumors and chloroquine efficacy. Proc. Natl Acad. Sci. USA 113, 182-187 (2016).

111. Guo, J. Y. et al. Activated Ras requires autophagy to maintain oxidative metabolism and tumorigenesis. Genes Dev. 25, 460-470 (2011).

112. Hashimoto, D. et al. Autophagy is needed for the growth of pancreatic adenocarcinoma and has a cytoprotective effect against anticancer drugs. Eur. J. Cancer 50, 1382-1390 (2014).

113. Wolpin, B. M. et al. Phase II and pharmacodynamic study of autophagy inhibition using hydroxychloroquine in patients with metastatic pancreatic adenocarcinoma. Oncologist 19, 637-638 (2014).

114. Macintosh, R. L. \& Ryan, K. M. Autophagy in tumour cell death. Semin. Cancer Biol. 23, 344-351 (2013).

115. Jenks, S. AACR highlights: promise for treating pancreatic cancer. J. Natl Cancer Inst. 103, 786-787 (2011)

116. Gomez, V. E., Giovannetti, E. \& Peters, G. J. Unraveling the complexity of autophagy: potential therapeutic applications in pancreatic ductal adenocarcinoma. Semin. Cancer Biol. 35, 11-19 (2015).

117. US National Library of Medicine. ClinicalTrials.gov, https://clinicaltrials.gov/ct2/show/NCT01494155? term $=01494155$ \&rank $=1$ (2011).

118. US National Library of Medicine. ClinicalTrials.gov, https://clinicaltrials.gov/ct2/show/NCT01506973? term $=01506973$ \&rank $=1$ (2012)

119. US National Library of Medicine. ClinicalTrials.gov, https://clinicaltrials.gov/ct2/show/NCT01128296? term $=01128296$ drank $=1$ (2010)

120. US National Library of Medicine. ClinicalTrials.gov https://clinicaltrials.gov/ct2/show/NCT01978184? term $=01978184 \&$ rank $=1$ (2013)

121. US National Library of Medicine. ClinicalTrials.gov, https://clinicaltrials.gov/ct2/show/NCT01777477? termNCT01777477\&rank = 1 (2013).

122. Moore, B. R et al. Pharmacokinetics, pharmacodynamics, and allometric scaling of chloroquine in a murine malaria model. Antimicrob. Agents Chemother. 55, 3899-3907 (2011).

123. Adelusi, S. A. \& Salako, L. A. Tissue and blood concentrations of chloroquine following chronic administration in the rat. J. Pharm. Pharmacol. 34, 733-735 (1982)

124. Bajpai, A. K. \& Choubey, J. Design of gelatin nanoparticles as swelling controlled delivery system for chloroquine phosphate. J. Mater. Sci. Mater. Med. 17, 345-358 (2006)

125. Joshi, P. et al. The anticancer activity of chloroquinegold nanoparticles against MCF-7 breast cancer cells. Colloids Surf. B Biointerfaces 95, 195-200 (2012). 
126. Adiseshaiah, P. P. et al. Synergistic combination therapy with nanoliposomal C6-ceramide and vinblastine is associated with autophagy dysfunction in hepatocarcinoma and colorectal cancer models. Cancer Lett. 337, 254-265 (2013).

127. Rangwala, R. et al. Combined MTOR and autophag inhibition: phase I trial of hydroxychloroquine and temsirolimus in patients with advanced solid tumors and melanoma. Autophagy 10, 1391-1402 (2014).

128. Klein, K. et al. Role of TFEB-driven autophagy regulation in pancreatic cancer treatment. Int. J. Oncol. 49, 164-172 (2016)

129. Pardo, R. et al. Gemcitabine induces the VMP1-mediated autophagy pathway to promote apoptotic death in human pancreatic cancer cells. Pancreatology 10, 19-26 (2010).

130. Cowan, R. W. \& Maitra, A. Genetic progression of pancreatic cancer. Cancer J. 20, 80-84 (2014)

131. Rucki, A. A. \& Zheng, L. Pancreatic cancer stroma: understanding biology leads to new therapeutic strategies. World J. Gastroenterol. 20, 2237-2246 (2014).

132. Duner, S., Lopatko Lindman, J., Ansari, D., Gundewar, C. \& Andersson, R. Pancreatic cancer: the role of pancreatic stellate cells in tumor progression. Pancreatology 10, 673-681 (2010).

133. Provenzano, P. P. et al. Enzymatic targeting of the stroma ablates physical barriers to treatment of pancreatic ductal adenocarcinoma. Cancer Cell 21. 418-429 (2012)

134. Jacobetz, M. A. et al. Hyaluronan impairs vascular function and drug delivery in a mouse model of pancreatic cancer. Gut 62, 112-120 |(2013).

135. Cassidy, J. W., Caldas, C. \& Bruna, A. Maintaining tumor heterogeneity in patient-derived tumo xenografts. Cancer Res. 75, 2963-2968 (2015)

136. Choi, S. Y. et al. Lessons from patient-derived xenografts for better in vitro modeling of human cancer. Adv. Drug Deliv. Rev. 79-80, 222-237 (2014).

137. Mak, I. W., Evaniew, N. \& Ghert, M. Lost in translation: animal models and clinical trials in cancer treatment. Am. J. Transl Res. 6, 114-118 (2014).

38. Ozdemir, B. C. et al. Depletion of carcinomaassociated fibroblasts and fibrosis induces immunosuppression and accelerates pancreas cancer with reduced survival. Cancer Cell 25, 719-734 (2014).

139. De Jesus-Acosta, A. et al. A phase Il study of vismodegib, a hedgehog $(\mathrm{Hh})$ pathway inhibitor, combined with gemcitabine and nab-paclitaxel (nab-P) in patients (pts) with untreated metastatic pancreatic ductal adenocarcinoma (PDA) [abstract 257]. J. Clin. Oncol. 32 (2014).

140. Diop-Frimpong, B., Chauhan, V. P., Krane, S. Boucher, Y. \& Jain, R. K. Losartan inhibits collagen I synthesis and improves the distribution and efficacy of nanotherapeutics in tumors. Proc. Natl Acad. Sci. USA 108, 2909-2914 (2011).

141. Lee, J. J. et al. Stromal response to Hedgehog signaling restrains pancreatic cancer progression. Proc. Natl Acad. Sci. USA 111, E3091-E3100 (2014).

142. Bever, K. M. et al. The prognostic value of stroma in pancreatic cancer in patients receiving adjuvant therapy. HPB (Oxford) 17, 292-298 (2015).

143. US National Library of Medicine. ClinicalTrials.gov, https://clinicaltrials.gov/ct2/show/NCT01839487? term $=$ NCT01839487\&rank $=1$ (2013)

144. Hingorani, S. R. et al. High response rate and PFS with PEGPH20 added to nab-paclitaxel/gemcitabine in stage IV previously untreated pancreatic cancer patients with high-HA tumors: interim results of a randomized phase II study [abstract 4006]. J. Clin. Oncol. 33 (2015).

145. Chauhan, V. P. \& Jain, R. K. Strategies for advancing cancer nanomedicine. Nat. Mater. 12, 958-962 (2013).

146. Halford, S. et al. A phase II study evaluating the tolerability and efficacy of CAELYX (liposomal doxorubicin, Doxil) in the treatment of unresectable pancreatic carcinoma. Ann. Oncol. 12, 1399-1402 (2001).

147. Ma, W. W. \& Hidalgo, M. The winning formulation: the development of paclitaxel in pancreatic cancer Clin. Cancer Res. 19, 5572-5579 (2013).

148. Nakai, Y. et al. Inhibition of renin-angiotensin system affects prognosis of advanced pancreatic cancer receiving gemcitabine $\mathrm{Br}$.J. Cancer 103, 1644-1648 (2010).

149. US National Library of Medicine. ClinicalTrials.gov, https://clinicaltrials.gov/ct2/show/NCT01821729? term $=$ NCT01821729\&rank = 1 (2013).

150. Jain, R. K. \& Stylianopoulos, T. Delivering nanomedicine to solid tumors. Nat. Rev. Clin. Oncol. 7 , 653-664 (2010).

151. Boucher, Y., Baxter, L. T. \& Jain, R. K. Interstitial pressure gradients in tissue-isolated and subcutaneous tumors: implications for therapy. Cancer Res. 50, 4478-4484 (1990).

152. Chauhan, V. P. et al. Normalization of tumour blood vessels improves the delivery of nanomedicines in a size-dependent manner. Nat. Nanotechnol. 7, 383-388 (2012).

153. Chen, N. et al. Pharmacokinetics and pharmacodynamics of nab-paclitaxel in patients with solid tumors: disposition kinetics and pharmacology distinct from solvent-based paclitaxel. J. Clin. Pharmacol. 54, 1097-1107 (2014).

154. Shea, J. E., Nam, K. H., Rapoport, N. \& Scaife, C. L. Genexol inhibits primary tumour growth and metastases in gemcitabine-resistant pancreatic ductal adenocarcinoma. HPB (Oxford) 13, 153-157 (2011).

155. Saif, M. W. et al. Phase II clinical trial of paclitaxel loaded polymeric micelle in patients with advanced pancreatic cancer. Cancer Invest. 28, 186-194 (2010).

156. US National Library of Medicine. ClinicalTrials.gov, https://clinicaltrials.gov/ct2/show/NCT02739633 (2013).

157. Saito, Y., Yasunaga, M., Kuroda, J., Koga, Y. \& Matsumura, Y. Enhanced distribution of NK012. a polymeric micelle-encapsulated $\mathrm{SN}-38$, and sustained release of $\mathrm{SN}-38$ within tumors can beat a hypovascular tumor. Cancer Sci. 99, 1258-1264 (2008)

158. Cabral, H. et al. Accumulation of sub-100 nm polymeric micelles in poorly permeable tumours depends on size. Nat. Nanotechnol. 6, 815-823 (2011).

159. Kano, M. R. et al. Improvement of cancer-targeting therapy, using nanocarriers for intractable solid tumors by inhibition of TGF- $\beta$ signaling. Proc. Natl Acad. Sci. USA 104, 3460-3465 (2007)

160. Kano, M. R. et al. Comparison of the effects of the kinase inhibitors imatinib, sorafenib, and transforming growth factor- $\beta$ receptor inhibitor on extravasation of nanoparticles from neovasculature. Cancer Sci. 100. 173-180 (2009).

161. Tong, R. T. et al. Vascular normalization by vascular endothelial growth factor receptor 2 blockade induces a pressure gradient across the vasculature and improves drug penetration in tumors. Cancer Res. 64 3731-3736 (2004)

162. Jain, R. K. Normalization of tumor vasculature: an emerging concept in antiangiogenic therapy. Science 307, 58-62 (2005)

163. Sahora, K. et al. A phase II trial of two durations of Bevacizumab added to neoadjuvant gemcitabine for borderline and locally advanced pancreatic cancer. Anticancer Res. 34, 2377-2384 (2014).

164. Tian, W. et al. Efficacy and safety profile of combining agents against epidermal growth factor receptor or vascular endothelium growth factor receptor with gemcitabine-based chemotherapy in patients with advanced pancreatic cancer: a meta-analysis. Pancreatology $13,415-422$ (2013).

165. Crane, C. H. et al. Phase II study of bevacizumab with concurrent capecitabine and radiation followed by maintenance gemcitabine and bevacizumab for locally advanced pancreatic cancer: Radiation Therapy Oncology Group RTOG 0411. J. Clin. Oncol. 27 4096-4102 (2009)

166. Baxter, L. T. \& Jain, R. K. Transport of fluid and macromolecules in tumors. IV. A microscopic model of the perivascular distribution. Microvasc. Res. 41 , 252-272 (1991).

167. Lieleg, O., Baumgartel, R. M. \& Bausch, A. R. Selective filtering of particles by the extracellular matrix: an electrostatic bandpass. Biophys. J. 97, 1569-1577 (2009).

168. Stylianopoulos, T. et al. Diffusion of particles in the extracellular matrix: the effect of repulsive electrostatic interactions. Biophys. J. 99, 1342-1349 (2010)

169. Hrkach, J. et al. Preclinical development and clinical translation of a PSMA-targeted docetaxel nanoparticle with a differentiated pharmacological profile. Sci. Transl Med. 4, $128 \mathrm{ra39}$ (2012).
170. Sugahara, K. N. et al. Tissue-penetrating delivery of compounds and nanoparticles into tumors. Cancer Cell $16,510-520$ (2009)

171. Garrido-Laguna, I. \& Hidalgo, M. Pancreatic cancer: from state-of-the-art treatments to promising novel therapies. Nat. Rev. Clin. Oncol. 12, 319-334 (2015).

172. Chauhan, V. P. et al. Compression of pancreatic tumor blood vessels by hyaluronan is caused by solid stress and not interstitial fluid pressure. Cancer Cell 26, 14-15 (2014)

173. Gerlowski, L. E. \& Jain, R. K. Microvascular permeability of normal and neoplastic tissues. Microvasc. Res. 31, 288-305 (1986).

174. Matsumura, Y. \& Maeda, H. A new concept for macromolecular therapeutics in cancer chemotherapy: mechanism of tumoritropic accumulation of proteins and the antitumor agent smancs. Cancer Res. 46 6387-6392 (1986).

175. Adiseshaiah, P. P., Hall, J. B. \& McNeil, S. E. Nanomaterial standards for efficacy and toxicity assessment. Wiley Interdiscip. Rev. Nanomed. Nanobiotechnol. 2, 99-112 (2010)

176. Phillips, P. A. et al. Cell migration: a novel aspect of pancreatic stellate cell biology. Gut 52, 677-682 (2003).

177. Li, J., Wientjes, M. G. \& Au, J. L. Pancreatic cancer: pathobiology, treatment options, and drug delivery. AAPS J. 12, 223-232 (2010).

178. Dobrovolskaia, M. A. Pre-clinical immunotoxicity studies of nanotechnology-formulated drugs: Challenges, considerations and strategy. J. Control Release 220, 571-583 (2015)

179. Dobrovolskaia, M. A. \& McNeil, S. E. Immunological properties of engineered nanomaterials. Nat. Nanotechnol. 2, 469-478 (2007).

180. McNeil, S. E. Nanoparticle therapeutics: a personal perspective. WIRES Nanomed. Nanobiotechnol. 1 264-271 (2009)

181. Ramanathan R. K et al A phase I dose escalation study of TKM-080301, a RNAi therapeutic directed against PLK1, in patients with advanced solid tumors [abstract LB-289]. Cancer Res. 73 (2013).

182. Mahalingam, D. et al. Phase I study of intravenously administered ATI-1123, a liposomal docetaxel formulation in patients with advanced solid tumors. Cancer Chemother. Pharmacol. 74, 1241-1250 (2014).

183. Mita, M. et al. A phase 1 study of BIND-014, a PSMA-targeted nanoparticle containing docetaxel, administered to patients with refractory solid tumors on a weekly schedule [abstract CT210]. Cancer Res. 74 (2014)

184. Deeken, J. F. et al. A phase I study of liposomalencapsulated docetaxel (LE-DT) in patients with advanced solid tumor malignancies. Cancer Chemother. Pharmacol. 71, 627-633 (2013)

185. NanoCarrier. Results of Asia phase I/II clinical study of NC-6004Nanoplatin ${ }^{\circledR}$ presented at Japanese Cancer Association Meeting. http://pdf.irpocket.com/C4571/ GpH7/xajp/i7aV.pdf (2013).

\section{Acknowledgements}

The authors thank Dr Stephan T. Stern and Dr Jennifer H. Grossman for their critical review of the manuscript, and Allen Kane of Leidos Biomedical Research for assistance with graphic illustrations. The work of P.P. A. R.M.C., and S.E.M. has been funded, in whole or in part, by Federal funds from the Frederick National Laboratory for Cancer Research, US $\mathrm{NIH}$, under contract HHSN261200800001E. The content of this publication does not necessarily reflect the views or policies of the Department of Health and Human Services, nor does mention of trade names, commercial products or organizations imply endorsement by the US Government.

\section{Author contributions}

P.P.A researched the data for the article and wrote the manuscript. RM.C assisted with drafting of the manuscript. All authors contributed to discussions of content and reviewed and/or edited manuscript before submission.

Competing interests statement

P.P.A., R.M.C, and S.E.M. are employees of Leidos Biomedical Research the operations and technical support contractor for the Frederick National Laboratory for Cancer Research. S.S.H. declares no competing interests.

FURTHER INFORMATION ClinicalTrials.gov: https://www.clinicaltrials.gov

ALL LINKS ARE ACTIVE IN THE ONLINE PDF 